\title{
Selected results from the ISUAL/FORMOSAT2 mission
}

\author{
Rue-Ron $\mathrm{Hsu}^{1}$, Han-Tzong Su${ }^{1, *}$, Alfred Bing-Chih Chen ${ }^{2}$, and Cheng-Ling Kuo ${ }^{3}$ \\ ${ }^{1}$ Department of Physics, National Cheng Kung University, Tainan City, Taiwan \\ ${ }^{2}$ Institute of Space and Plasma Sciences, National Cheng Kung University, Tainan City, Taiwan \\ ${ }^{3}$ Institute of Space Science, National Central University, Taoyuan City, Taiwan
}

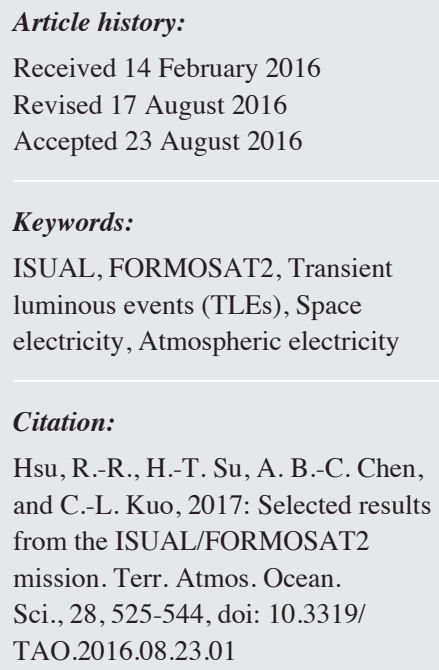

\begin{abstract}
The ISUAL (Imager of Sprites and Upper Atmospheric Lightning) is a scientific payload onboard the FORMOSAT2 satellite (FS2). This the first satellite project survey of global transient luminous events (TLEs) as one of the mission objectives. Since the ISUAL/FS2 launch in 2004, ISUAL has continuously monitored TLE occurrences over pre-midnight regions in the past ten plus years. As of December 2015, more than forty thousand TLEs, including the sub-species like elves, sprites, spritehalos, blue jets and gigantic jets, have been recorded from this space platform. In the meantime, as the supporting facilities to the space-borne ISUAL experiment, ground optical imagery systems have been deployed to observe TLEs occurring near Taiwan with several radio wave detection ground stations also installed to register lightningor TLE-related radio signals called sferics. From analyzing the observed events and the associated sferics, many TLE characteristics have been inferred. Among the most notable factors are the global distributions, the occurrence rates, and the charge injection and the energy impact to the upper atmosphere. In this article, the ISUAL payload, the auxiliary ground facilities and a few selected TLE survey results from the ISUAL mission are concisely presented.
\end{abstract}

\section{INTRODUCTION}

Eyewitness reports of fleeting luminous phenomena above active thunderstorms have circulated around the scientific community for over a century (Lyons et al. 2000). One exemplifying recount of a possible jet event occurring near Taiwan was given by a pilot "...I was co-pilot on a DC8 freighter flying from Fukuoka-Japan to Hong Kong. It was the evening of October 10th 1979. I was fascinated by a particularly active area of thunderstorms close to, or possibly over the east coast of Taiwan. The area was probably 40 miles by 20 miles, showing a solid return on our weather radar. There were somewhere between 12 and 20 active cells, which meant an almost continuous illumination of the area from the lightning discharges, maybe 2 or 3 each second. I then saw a solitary lightning bolt come out of the top of this mass and disappear into space. It split into several branches and the color changed from bright white to blue and purple and appeared to thin into nothing..." (Mark Tanner 2004, private communication). This

\footnotetext{
* Corresponding author

E-mail:htsu@ncku.edu.tw
}

report gives a very precise setting for the occurrence of an upward discharge from the cloud-top and some of its optical characteristics. However, with no image record to support this type of reports, they were often ignored by the atmospheric electricity community. One of them was from C. T. R. Wilson (Williams 2010), a Nobel Prize winning physicist of the cloud chamber fame. The study of transient luminous events (TLEs) only became a legitimated and very active research field after the serendipitous discovery of sprites in 1989 (Franz et al. 1990).

Right after the discovery of sprites, it was informally termed and perceived as "cloud-to-space lightning"; a name that aroused concerns that this newly discovered upward lightning may pose a threat to spacecraft during the launch and recovery phases. The urgency to clarify the occurrence frequency of sprites and their basic characteristics soon lead to intensive ground campaigns around the globe. In short succession, additional thundercloud-top luminous phenomena were found to include elves, sprite halos, blue starters, blue jets as well as gigantic jets (Pasko 2010; Pasko et al. 2012 and the references therein). They are collectively 
termed "transient luminous events" (TLEs; Fig. 1). TLE can be broadly divided into two groups: lightning-induced events like sprites, elves and sprite halos and the second class is upward discharge between a thundercloud and the lower ionosphere like blue starters, blue jets and gigantic jets. More specifically, elves (Fukunishi et al. 1996) span the altitudes of $\sim 80-90 \mathrm{~km}$ and are induced by the intense lightning electromagnetic pulse (EMP). Sprites, with a predominately-red luminous structure that spans $\sim 40-90 \mathrm{~km}$ (Sentman and Wescott 1993), are produced by the residual quasi-static electric field following an exceptionally strong cloud-to-ground (CG) lightning (Pasko et al. 1997). Sprite halo, which shares the same generation mechanism with the sprite and centers around $70 \mathrm{~km}$ altitude (Barrington-Leigh et al. 2001), can appear alone or accompanied by a sprite. Blue starters, blue jets and gigantic jets are upward electric discharges between the thunderclouds and the ionosphere (Wescott et al. 1995; Pasko et al. 2002; Su et al. 2003) and are considered the counterparts of CG lightning. Among the electric jets, blue starters extend from the cloud-top to $\sim 25 \mathrm{~km}$. Blue jets can reach $40 \mathrm{~km}$ (Wescott et al. 1995), while gigantic jets terminate at the lower ionosphere boundary hence has the highest terminal altitude.

During the ground TLE campaigns it was soon realized that the local weather and terrain around the observation site often are the deciding factors for the success or failure of a campaign. The field-of-view depth for TLE observation is typically less than $800 \mathrm{~km}$, even under ideal conditions. More importantly, when it comes to spectroscopic measurements, the intervene atmosphere between the TLEs and the observational instruments is an insurmountable obstacle that wipes out some of the critical TLE spectral information. To alleviate the confinement of local weather, local terrain and the atmospheric absorption of TLE emissions, carrying out observations from a space platform became a highly desirable option that sent many proposal writings in motion with the ISUAL mission as one of them.

The ISUAL payload was proposed in 1998 to the National Space Organization in Taiwan to compete for the only scientific payload opportunity on the FORMOSAT2 satellite, previously also known as the ROCSAT-2 satellite. After a rigorous selection process, the contract was awarded to the ISUAL team at the end of 1998. Therefore, the ISUAL payload became the first satellite mission to carry out a long term survey of the then newly discovered TLEs from 891$\mathrm{km}$ low earth sun-synchronous orbits (Chern et al. 2003). Among the mission goals ISUAL seeks to determine the global distribution and occurrence rate of TLEs as well as
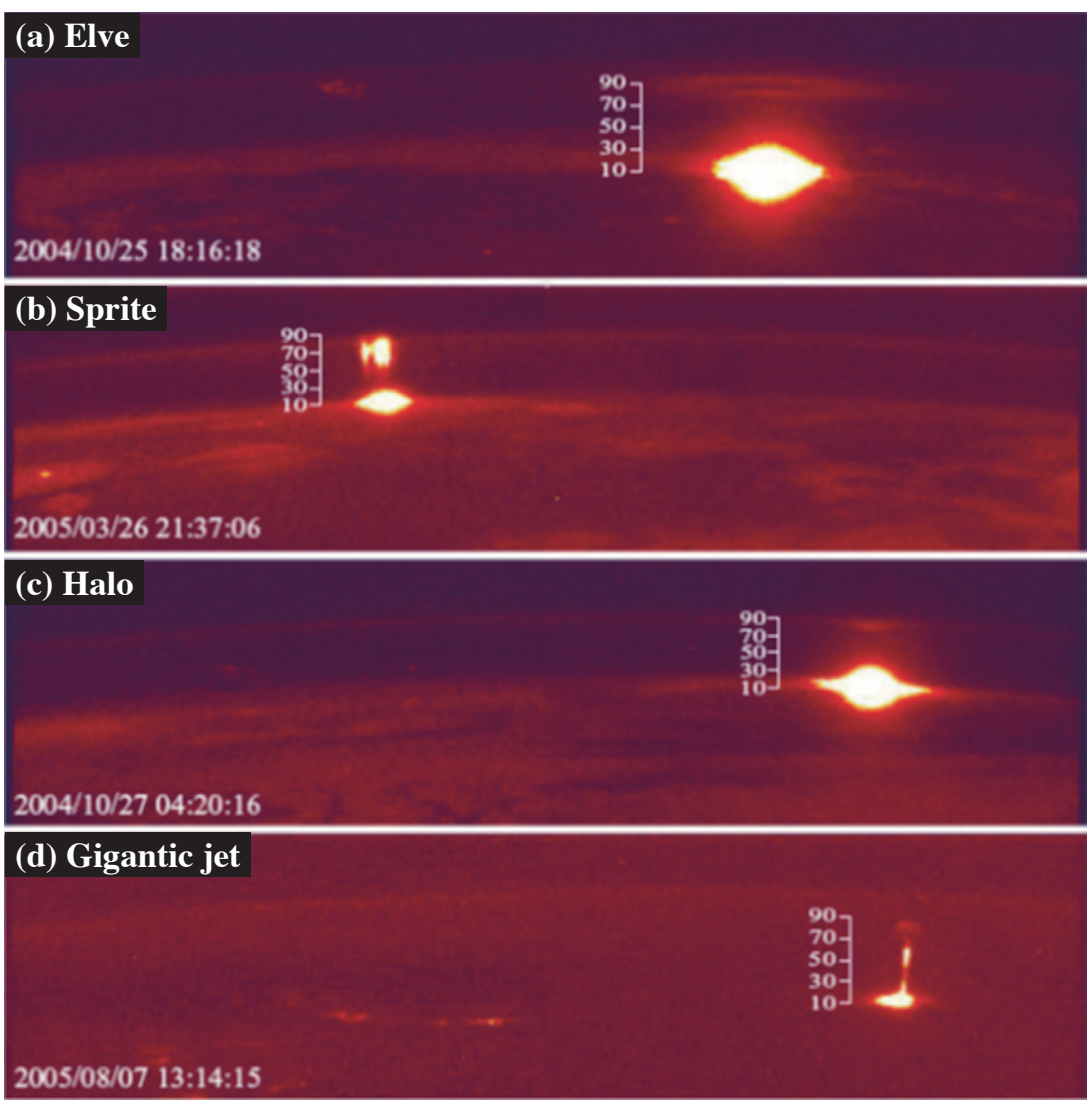

Fig. 1. Representative TLEs from the ISUAL experiment: (a) an elve, (b) a sprite, (c) a halo, and (d) a gigantic jet. The images are in false colors and the colors carry no scientific meaning. (Color online only) 
to infer the spatial, temporal and spectral properties of the thunderstorm-related transient luminous phenomena.

Since 2001, as an auxiliary program for the ISUAL mission, the NCKU ISUAL team has routinely staged summer ground TLE campaigns in Taiwan and obtained some good results. For examples see Hsu et al. (2003), Chou et al. (2011), and Huang et al. (2012). After entering the satellite observation phase, between the first light in July 2004 and the end of 2015 ISUAL has recorded more than forty thousand TLEs from orbit. Though analyzing TLE events observed in the ground and in the space-borne ISUAL observations as well as the associated data recorded by the ground radio-band stations, important TLE characteristics have been inferred. A few selected results from the ISUAL mission are reported. After a brief introduction to the ISUAL and auxiliary ground instruments, the global distribution, the occurrence rate and the physical characteristics of the TLEs from the ISUAL mission are reported. A summary of the ISUAL mission is given as a conclusion.

\section{THE INSTRUMENTS}

Most of the data reported in this article were recorded by three types of instruments: the ISUAL payload on the FORMOSAT satellite, the NCKU low-light-level ground imaging systems, and the NCKU radio-signal recording systems.

\subsection{The ISUAL Payload}

The ISUAL (Chern et al. 2003) is mounted on the payload deck of the FORMOSAT2 satellite and contains three sensor packages: an intensified CCD imager (Imager) with a $20^{\circ}(\mathrm{H}) \times 5^{\circ}(\mathrm{V})$ field of view, a six-channel spectrophotometer (SP), and a dual-band array photometer (AP); Fig. 2.
The ISUAL iCCD imager is equipped with a rotatable filter wheel, with filters covering the emission band systems of $\mathrm{N}_{2}, \mathrm{O}_{2}$ and oxygen atoms; the filter selection includes filter 1 (623 - $750 \mathrm{~nm})$, filter 2 (762 nm; $758-769 \mathrm{~nm})$, filter 3 $(630 \mathrm{~nm} ; 626-633 \mathrm{~nm})$, filter $4(427.8 \mathrm{~nm} ; 425-432 \mathrm{~nm})$, filter 5 (557.7 nm; 555 - $563 \mathrm{~nm})$, and filter 6 (open). Filter 1 is used in most of the routine TLE survey.

The ISUAL SP is bore-sighted with the ISUAL imager. It contains six channels that cover the major emission bands of molecular nitrogen including SP1 (150 - $290 \mathrm{~nm}$; FUV; $\mathrm{N}_{2}$ LBH band), SP2 (337.1 nm; 333.5 - $341.2 \mathrm{~nm}$; for $2 \mathrm{PN}_{2} 0$ 0), SP3 (391.4 nm; $387.1-393.6 \mathrm{~nm}$; for $1 \mathrm{NN}_{2}{ }^{+} 0$ - 0), SP4 (608.9 - $753.4 \mathrm{~nm}$; for $1 \mathrm{PN}_{2}$ band), SP5 (777.4 nm; 773.6 $784.7 \mathrm{~nm}$; for lightning OI emission), and SP6 (244 - $392 \mathrm{~nm}$; for $2 \mathrm{PN}_{2}$ band). As soon as the ISUAL is powered on, the SP continuously writes $1 \mathrm{~ms}$ resolution data to a circular buffer. With a valid event trigger, the data between $24 \mathrm{~ms}$ prior to the trigger and $181 \mathrm{~ms}$ posteriori to the trigger are stored.

The ISUAL AP consists of a blue (370 - $450 \mathrm{~nm})$ module and a red (530 - $650 \mathrm{~nm})$ module. Each module contains an array of sixteen-vertically stacked PMT tubes. The combined FOV of AP is $22^{\circ}(\mathrm{H}) \times 3.7^{\circ}(\mathrm{V})$, which is slightly wider but having a smaller vertical FOV comparing to those for the ISUAL imager and SP. The AP sampling rate is $20 \mathrm{kHz}$ from $8 \mathrm{~ms}$ before an event trigger to $20 \mathrm{~ms}$ after and then slows down to $2 \mathrm{kHz}$ until $240 \mathrm{~ms}$ after the trigger. The ISUAL AP is designed to provide high temporal and medium spatial resolving photometric data.

For the TLE survey, when the ISUAL spectrophotometric channels 1, 2, 6 simultaneously exceed preset threshold levels an ISUAL trigger event is flagged. The ISUAL then snaps six consecutive image frames. Each frame has a typical exposure time of $29 \mathrm{~ms}$ with a $1 \mathrm{~ms}$ dead time for read-out. In the meantime, the associated SP and AP data are pulled from the circular buffers and saved.

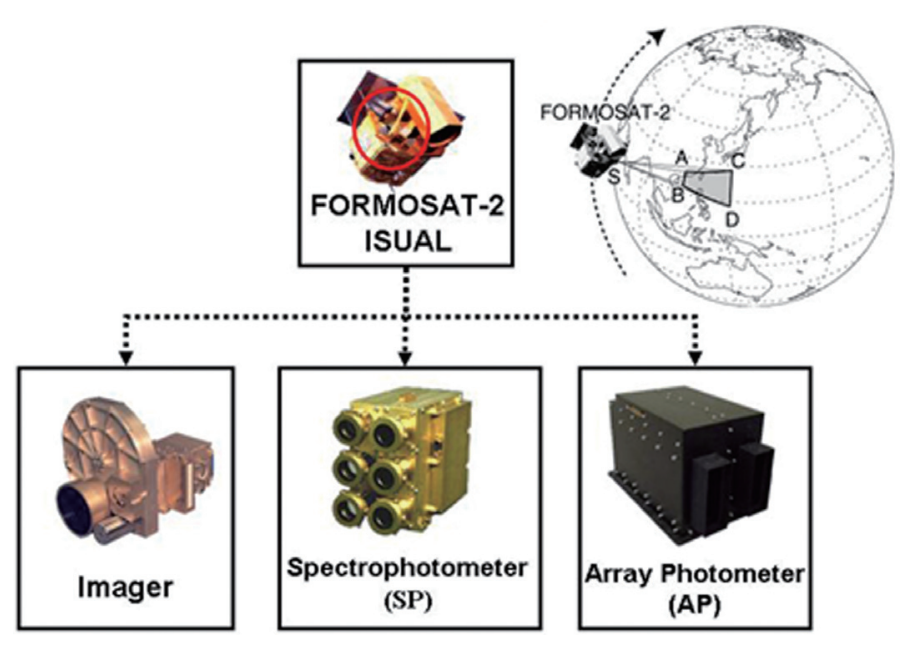

Fig. 2. The ISUAL payload on the FORMOSAT2 satellite and the sensor packages. The area surveyed by the ISUAL experiment is the pre-midnight region. (Color online only) 


\subsection{The NCKU Ground Imaging Systems}

The imaging systems used in our ground TLE campaigns usually consist of a low-light-level Watec CCD, a camera lens and a data recorder. The FOV of the imaging systems varies depending on the focal length of the lens. But it typically spans more than 30 degree in the lateral direction. Except for a few occasions, the imaging systems are mostly standard NTSC camera running at a 30 frames per second. Currently, the NCKU group operates four remote-controllable TLE imaging systems separately located at Jin-Cheng Junior High School, NCKU, National Penghu University of Science and Technology (NPU), and National Taitung University (NTTU); Fig. 3.

\subsection{The NCKU Radio-Signal Recording Stations}

The NCKU team operates three sets of radio-band recording stations, covering the ULF (ultra-low frequency), ELF/VLF (extremely/very low frequency), and the LF (low frequency) bands; Fig. 3. The magnetic ULF station is located at the Lulin Observatory in the Yushan National Park, Taiwan. The system is based on a pair of EMI-BF4 horizontal magnetic coils which are sensitive in the radio frequency band between 0.3 and $500 \mathrm{~Hz}$, with coils either orientated parallel $(\mathrm{H}$; north south) or perpendicular to (D; east west) the geomagnetic field. The NCKU ELF/VLF station contains a pair of QUASAR horizontal induction sensors and one vertical electric field sensor, which are sensitive in the radio frequency band of $1.5 \mathrm{~Hz}$ to $15 \mathrm{kHz}$ and $0.1 \mathrm{~Hz}$ to $100 \mathrm{kHz}$, respectively. The LF $(30-300 \mathrm{kHz})$ system is constructed by Professor Steve Cummer's group at the Duke University, USA. Both the NCKU ELF/VLF and the LF systems reside in the suburban area of the Tainan City.

\section{THE TLE OCCURRENCE AND THE DISTRIBUTION}

\subsection{First Sprites Observed Over the Asian Continent}

The first ever ground TLE campaign in Taiwan was conducted between April and August of 2001 as preparation work for the upcoming ISUAL experiment $(\mathrm{Su}$ et al. 2002; Hsu et al. 2003). The weather in Taiwan is heavily influenced in spring and summer by convective systems and typhoons. Hence in the first Taiwan TLE campaign, only 27 nights had clear conditions that allowed observations to be carried out. Sprites were observed on five evenings. The first TLEs observation over the Asian continent was achieved on the second night (30 April 2001) of the campaign from the Ali Mountain Weather Station (AMWS). Forty sprites were observed from Ali Mountain, produced by two moderate thunderstorms in eastern Guangdong Province, China, $\sim 600 \mathrm{~km}$ to the west of the observation site. From the AMWS site TLEs over China and the Pacific Ocean can be monitored. For the sprites observed in this campaign, the majority of land sprites occurred in clusters, whereas most of the oceanic sprites appeared individually.

\subsection{Gigantic Jets over a Tropical Depression}

Upward electrical discharges from thunderclouds called blue jets and blue starters were first observed during a 1993 aircraft TLE campaign (Wescott et al. 1995). However, due to their small vertical extension, that can be easily blocked by the thundercloud, ground TLE campaigns generally do not expect to see them except for events like the $\sim 70 \mathrm{~km}$-height gigantic blue jet observed by Pasko et al. (2002) in Puerto Rico.

In the second (2002) ground TLE campaign in Taiwan

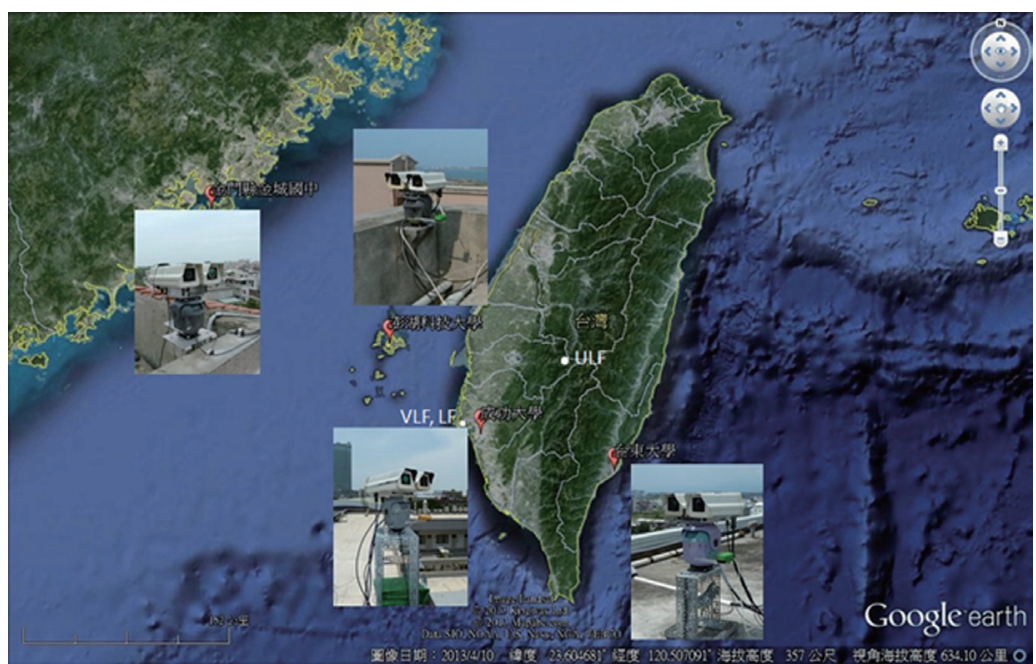

Fig. 3. The four remote-controllable TLE observation stations of the Taiwan TLE network. The stations are at NCKU, Jin-cheng JHS, NPU, and NTTU. The two white dots mark the locations of the NCKU ULF and VLF/LF stations, respectively. (Color online only) 
we continued to perform observations from the AMWS site but with very limited success. In the few days around 22 July 2002 when the AMWS was clouded over, we went to Kenting at the very southern tip of Taiwan to observe a low pressure system near Luzon Island, Philippines. On the evening of 22 July 2002, five gigantic jets that linked the cloud-top at $\sim 16 \mathrm{~km}$ elevation and the local ionosphere near $\sim 90 \mathrm{~km}$ altitude were seen to protrude from this system ( $\mathrm{Su}$ et al. 2003). The spatial development of these gigantic jets can be divided into three stages: the leading jet, the fully-developed jet, and the trailing jet. At the fully developed stage, two gigantic jets presented a tree-shaped upper part and a bluejet like lower section, and hence are called 'tree' jets. For the other three events the upper section looked like a carrot, the lower section resembled a blue jet and these are called 'carrot' jets; Fig. 4. From the associated radio band data it was inferred that these gigantic jets are negative cloud-to- ionosphere (-CI) discharges. Therefore, they would reduce the electrical potential between the ionosphere and ground and thus could affect the global electrical circuit.

\subsection{Classifications of Gigantic Jets}

Gigantic jets (GJs) actually can be classified into three types from their generating sequence and spectral properties (Chou et al. 2010). The generating sequence for the type I GJs resembles those reported in Su et al. (2003). After the fully-developed jet (FDJ) creates the discharge channel, the ISUAL photometers register a peak from a return-strokelike process. The ULF sferics of these type I GJs indicates that they are negative cloud-to-ionosphere discharges (-CIs). Type II GJs typically start as blue jets and then develop into GJs in $\sim 100 \mathrm{~ms}$; Fig. 5. Blue jets also often appeared at the same region before and after the type II GJs. We only had

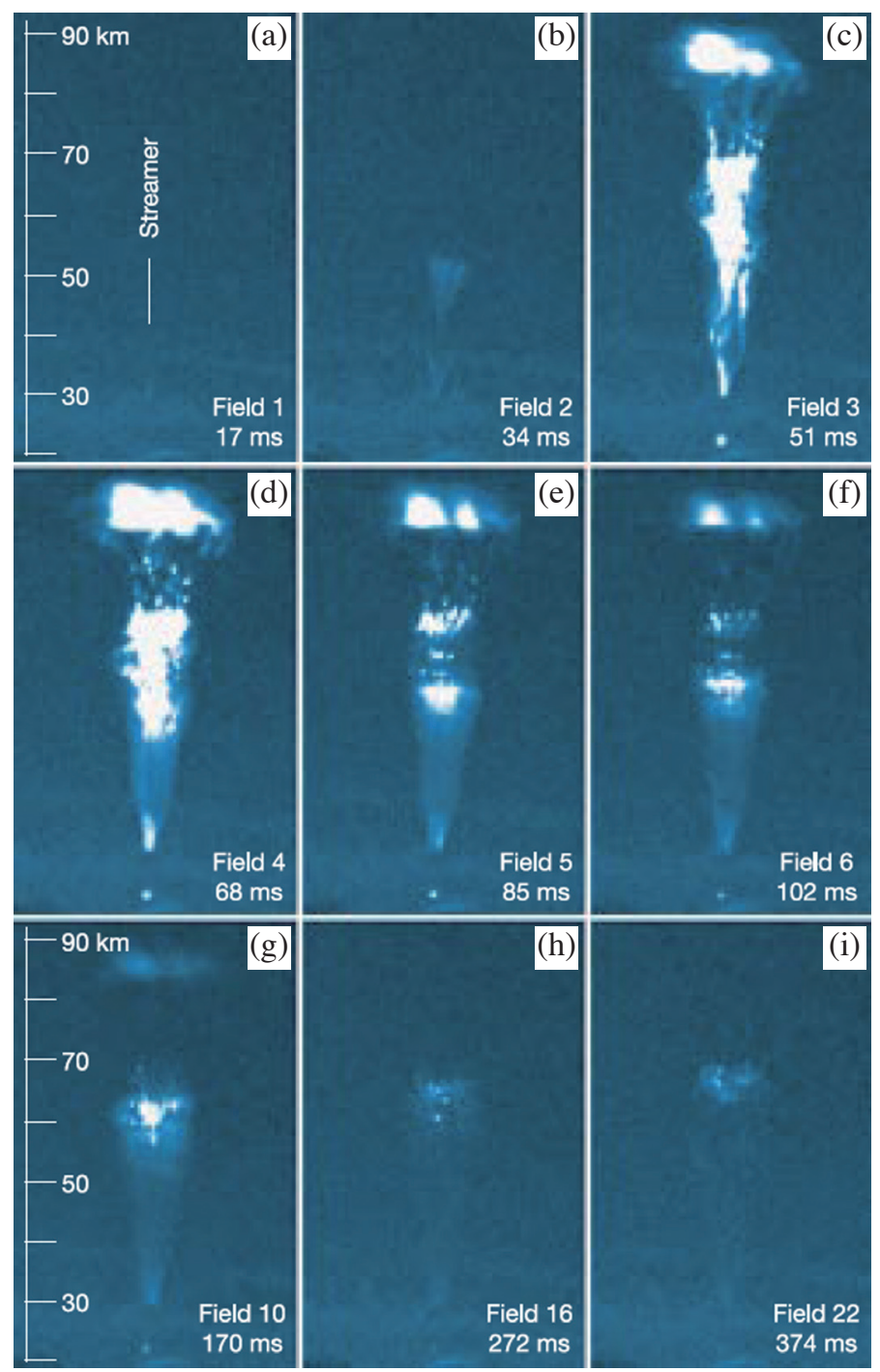

Fig. 4. A carrot-like gigantic jet observed on 22 July 2002. (a) - (b) The morphological evolution of this event contains the leading jet, (c) - (g) the fully-developed jet, and (h) - (i) the trailing jet. The luminous duration of the gigantic jet lasts for $\sim 650 \mathrm{~ms}$ (Su et al. 2003). (Color online only) 


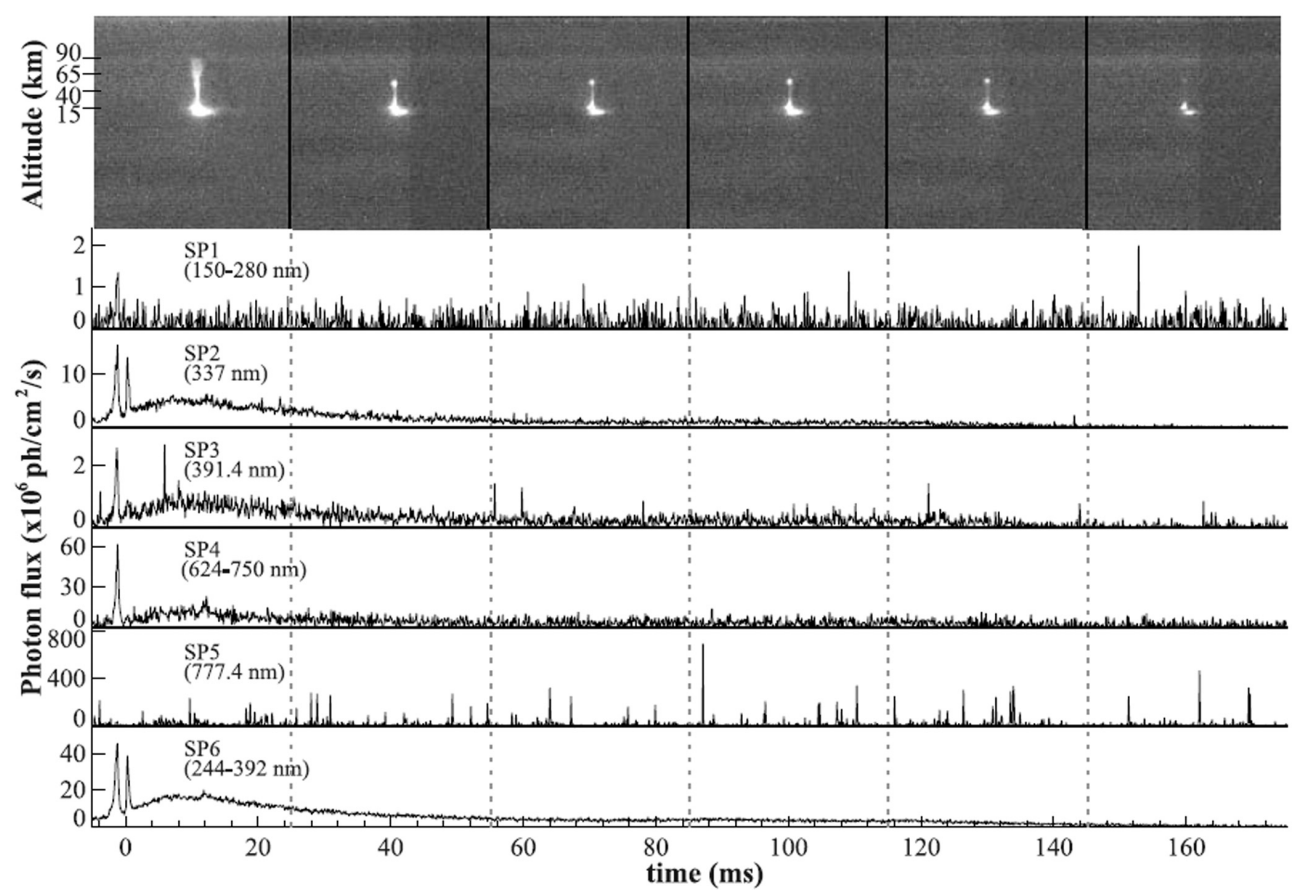

Fig. 5. A type II GJ recorded on 4 April 2009. The image sequence indicates that the development of this GJ started with a blue jet (Chou et al. 2010).

marginal evidence to support that the type II GJs are +CI discharge events but an independent study by Van der Velde et al. (2010) indeed proved it is a -CI-type event. Type III GJs were preceded by lightning and a GJ later occurred near this preceding lightning. The spectral data of the type III GJs are dominated by lightning emissions and the ULF data are noisy, thus both cannot be properly analyzed. However, the average brightness of the type III GJs is between those of the other two types of GJs. We believe that the discharge polarity of the type III GJs can either be negative or positive, depending critically on the type of the charge imbalance left by the trigger lightning.

\subsection{Electric Characteristics of the Gigantic Jets over Typhoon Lionrock}

From the NCKU TLE observation station, situated in the middle of Tainan City, Typhoon Lionrock was observed as it passed through southwest of the site at a closest distance of $\sim 210 \mathrm{~km}$. Due to the proximity of this severe weather system, more than a hundred TLEs were recorded over typhoon Lionrock on 31 August 2010. Huang et al. (2012) reported the results from the analysis of fourteen negative GJs from this system and their associated radio signals. These GJs are all found to be type-I GJs and thus are -CI discharges. Morphologically, these GJs have three forms: tree-like, carrotlike, and a new intermediate type called tree-carrot-like GJs. The ULF and ELF/VLF band sferics of these events contain features connected to GJ development stages, including the initiating lightning, the leading jet, the fully-developed jet, and the trailing jet. The key feature of the associated radio waveform for these GJs always includes a fast descending pulse linked to the GJ surge current, when they make the contact with the ionosphere. However, the detailed waveforms show a substantial variation. Based on the optical and radio frequency signals for these GJs, it is found that the tree-like GJs tend to have a relatively large surge current moment $(\mathrm{CM})(>60 \mathrm{kA} \mathrm{km})$ while the carrot-like and the treecarrot-like GJs both have a relatively small surge $\mathrm{CM}$ of less than $36 \mathrm{kA} \mathrm{km}$; Fig. 6. Meanwhile, a continuing $\mathrm{CM}$ of less than $27 \mathrm{kA} \mathrm{km}$ further separates the carrot-like GJs from the tree-carrot-like GJs. Also on the peak CM versus the charge moment change diagram for the initiating lightning, different subgroups of GJs exhibit different trends. This characteristics suggests that the forms of the negative GJs may have been determined at the initiating lightning stage.

\subsection{Secondary Jets and Secondary Gigantic Jets}

A secondary TLE is defined as a transient luminous event that occurs under a preceding sprite and then propagates from the cloud top toward the lower edge of the sprite or even beyond. Over the years ISUAL has detected several dozens of secondary TLEs (Fig. 7), Lee et al. (2012) studied the secondary jets and secondary GJs to elucidate their characteristics and their possible generating mechanisms. Among the analyzed events, secondary jets developed from the cloud top beneath preceding sprites and developed 


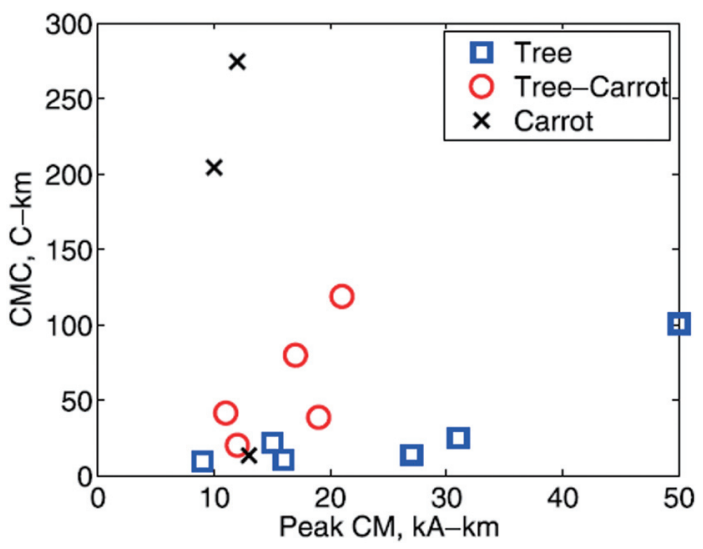

Fig. 6. The peak current moment (CM) versus the charge moment change (CMC) for the initiating lightning of 14 negative GJs (Huang et al. 2012). (Color online only)

(a)
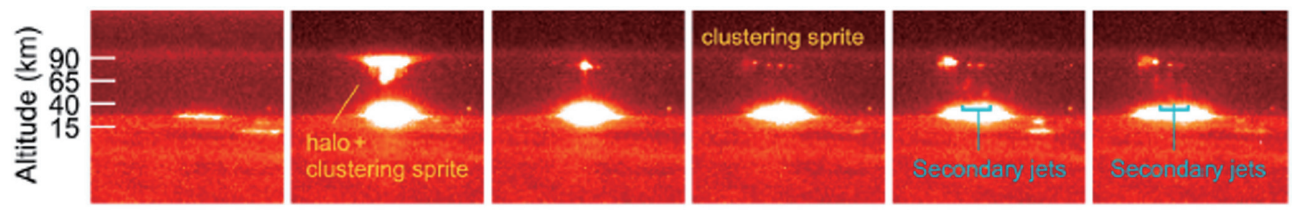

(b)
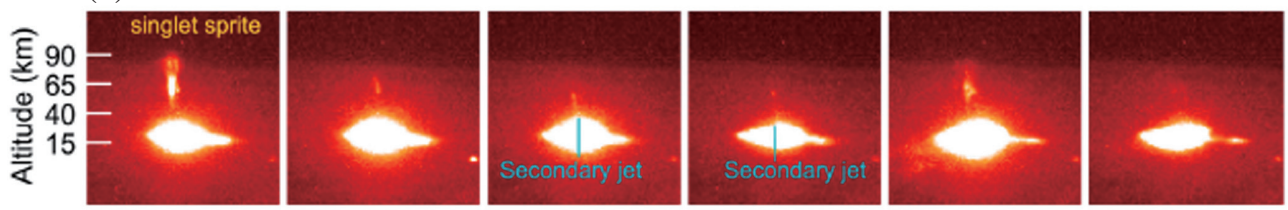

(c)
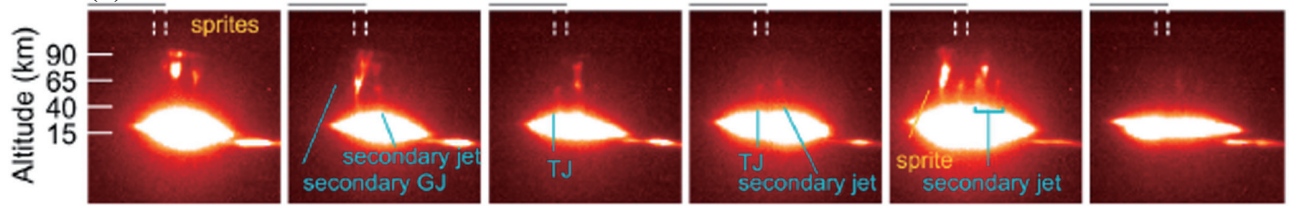

(d)
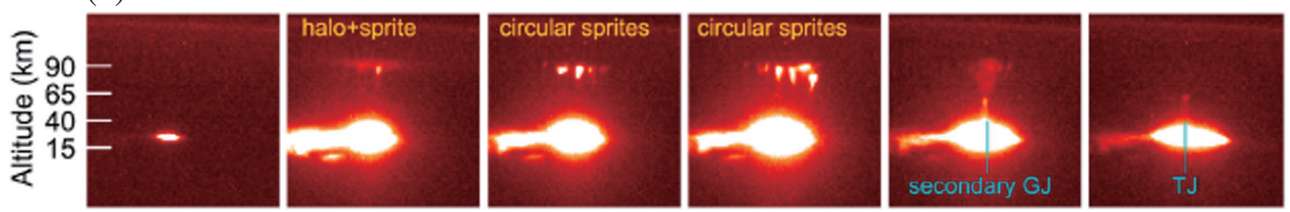

Fig. 7. Representative ISUAL secondary jets and GJs (cropped): (a) secondary jet 2010/05/31 23:27:48.128 UTC, (b) secondary jet 2005/07/19 14:57:27.065 UTC, (c) shifted secondary GJ 2009/06/29 23:19:47.958 UTC, and (d) pop-through secondary GJ 2008/09/30 21:52:39.660 UTC (Lee et al. 2012). (Color online only)

towards the lower edge of the sprites at $\sim 50 \mathrm{~km}$. The secondary jets contain vertically-oriented luminous columns and have a form resembling the trailing jet in a type-I GJ. The number of luminous columns in a secondary jet varies and seems to be affected by the size of the effective capacitor plate area near the base of the preceding sprites as well as the charge distribution left behind by the sprite-producing positive CG lightning. Similarly, secondary GJs also originated from the cloud top in the shielding area under the preceding sprites, and propagate upward to reach the lower ionosphere at $\sim 90 \mathrm{~km}$. Morphologically, the secondary GJs can either be a curvedly secondary GJ that extends outside the shielding area of the preceding sprite or be a straightcolumn secondary GJ that propagates through the center of the preceding circular distributed sprites. From examining the ISUAL imager data, it appears that the key in determining the terminal height of the secondary TLEs most likely is the local ionosphere boundary height established by the 
preceding sprites. The quantity and the dissemination of the negative charge in the thundercloud left behind by a spriteinducing positive CG discharge may have played critical roles in generating the secondary TLEs.

GJs between the cloud and the ionosphere plates are the counterparts of the conventional lightning between the cloud and the ground plates. Since +CG lightning is capable of producing sprites in the upper atmosphere, one may wonder could GJs induce sprites? Most of the GJs recorded by ISU$\mathrm{AL}$ do not seem to induce sprites, except for three secondary GJs in multi-TLE events. These events shared a similar generating sequence: each event started with a +CG-induced sprite, a secondary GJ followed within $\sim 30$ - $50 \mathrm{~ms}$, then a new sprite occurred near the GJ in $1 \mathrm{~ms}$ with no discernible associated impulsive lightning signal; Fig. 8. Cross-analyzing the spectral, imager and electromagnetic data of these three events, it was found that the new sprites are likely induced by the secondary GJs and the high current moment of the secondary GJs appears to be important for the induction of the new sprites (Lee et al. 2013). We suggest that these secondary GJs may have induced sprites, much as negative CGs do for the occurrence of negative sprites.

\subsection{Dim Elves and Their Detection}

The ISUAL far-ultraviolet (FUV) events are defined as valid ISUAL triggered events (Chang et al. 2010), which have no discernible TLE in the imager emissions but have substantial signal in the ISUAL FUV spectrophotometer channel (SP1). It has long been suspected that these FUV events could be dim TLEs. To rule out the possible contribution due to lightning, we performed lightning emission simulation and confirmed that the atmospheric absorption indeed prevents the lightning FUV emission from reaching the ISUAL sensors. Furthermore, the FUV emission of the FUV events as well as those of the elves all follow the lightning OI emission within $1 \mathrm{~ms}$. After analyzing the imager-N21P brightness as well as the FUV intensity of the elves, a linear relation similar to that reported in Kuo et al. (2007) was found, except having a different proportional constant; Fig. 9. The intensities of FUV events are similar to those of the dimmest elves and often is less than $1 \times 10^{4}$ photons $\mathrm{cm}^{-2}$. Putting all of this information together, it is concluded that the FUV events are dim elves eluding the detection of the ISUAL imager. Judging from the detection limits of the ISUAL spectrophotometer (SP) and the imager, for the before-the-limb elves the detection number from the SP is nearly 16 times higher than that of the imager. This result is consistent with a ratio of $\sim 13$ that was inferred from the U.S. National Lightning Detection Network (NLDN) peak current distribution for the negative cloud-to-ground lightning. Therefore, the ISUAL spectrophotometer can be used to perform elves survey, to infer the peak current of the elve-producing lightning and possibly be used to deduce other lightning parameters. Most importantly, this study implies that the imager-based elves survey could have undercounted the event number by an order of magnitude. Thus, in order to infer the correct elve global occurrence rate, one needs to apply a correction factor of ten or even more to the imager survey and the rate becomes 72 events minute ${ }^{-1}$.

\subsection{Occurrence of Elves During ENSO Events}

By comparing ISUAL/FORMOSAT2 elves, LISTRMM lightning, sea surface temperature (SST), and the El Niño southern oscillation (ENSO) between June 2005 and May 2010, we elucidated the impact of ENSO on the mesospheric elves and the tropospheric lightning ( $\mathrm{Wu}$ et al. 2012); Fig. 10. The studied areas were the ENSO-sensitive western Pacific, central Pacific and Tahiti regions, where a significant event anomaly is viewed as the impact flags of ENSO. The results support that the occurrence of elves shows un-mistakable responses to ENSO with a correlation coefficient greater than 0.6 for the coastal and the oceanic regions. The lightning occurrence is sensitive to the ENSO event in the oceanic regions, but the coastal regions have a low correlation to ENSO, due to the masking influence of the landmass. Therefore, between elves (elevated occurrence rate and its correlation with ENSO 0.64) and lightning (correlation 0.36) as indicators of ENSO, elves proved to have a wider applicable geographic range. Elves are induced by intense lightning; therefore the lightning response towards ENSO would be similar as that for elves. Hence, the intense lightning can also be used as an effective gauge of ENSO.

\subsection{Geographic Distribution of Elve-Producing Lightning}

It is a general assumption that intense lightning tends to have a higher efficiency in inducing TLEs. However, this conjecture has never been quantitatively tested. In this work (Chen et al. 2014), the lightning stroke data from the upgraded World Wide Lightning Location Network (WWLLN) and the ISUAL TLE data were used to investigate the energetics and geographic distribution of TLE-producing lightning. The results prove that the global median energy of the strikes that produced TLEs is at least one order of magnitude higher than the global median strike energy inferred from the WWLLN lightning; Fig. 11. Most interestingly, the elve-producing lightning energy distribution exhibits no oceanic and land disparity. These results indicate that elves are indeed triggered by energetic lightning and the energy of elve-inducing lightning is insensitive to the underlying landform. The spatial correlation between the ISUAL elves and the WWLLN lightning reveals that the geographic distribution of the ISUAL elves coincides with that for the most energetic $10 \%$ of WWLLN lightning 


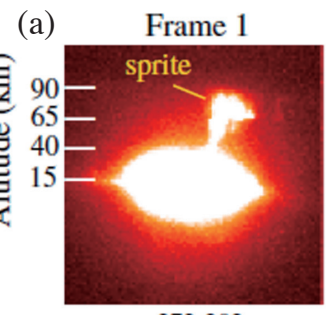

273-302
Frame 2

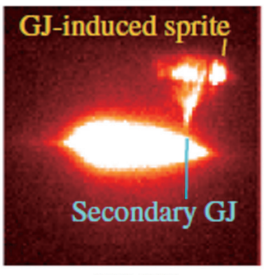

303-332
Time from 14:40:04 UT (ms)

Frame 3

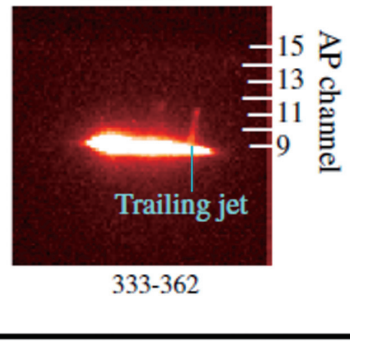

(c) Array Photometer (AP)

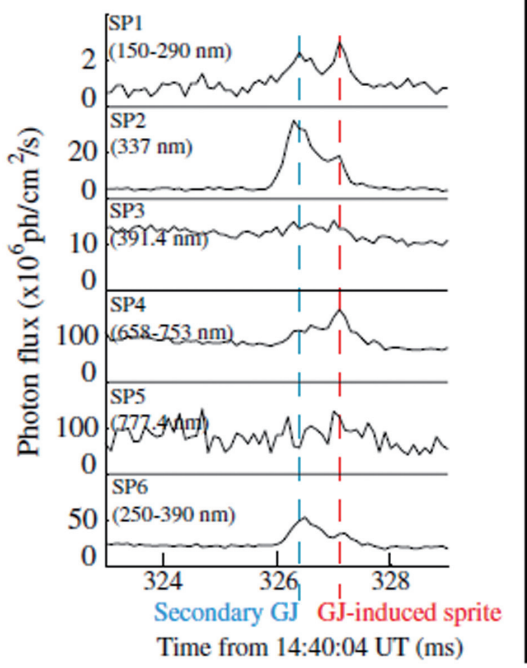
blue band $(370-450 \mathrm{~nm})$

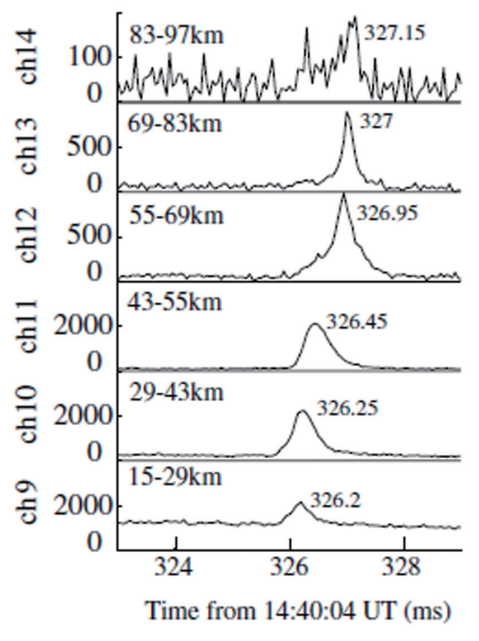

Fig. 8. Cropped image sequence and photometric signals for GJ 2008/09/30 21:52:39.660 UTC. (a) The images were recorded by the ISUAL imager through a 1PN2 $(623-750 \mathrm{~nm}$ ) filter. (b) The corresponding spectrophotometer data for the interval of 323 - 329 ms (relative to 14:40:04.000 UTC), which contain emissions from the GJ and the GJ-induced sprite. (c) The array photometer blue module data for the interval 323 - 329 ms; only the channels covering the altitudes of the GJ and the sprites are shown (Lee et al. 2013). (Color online only)

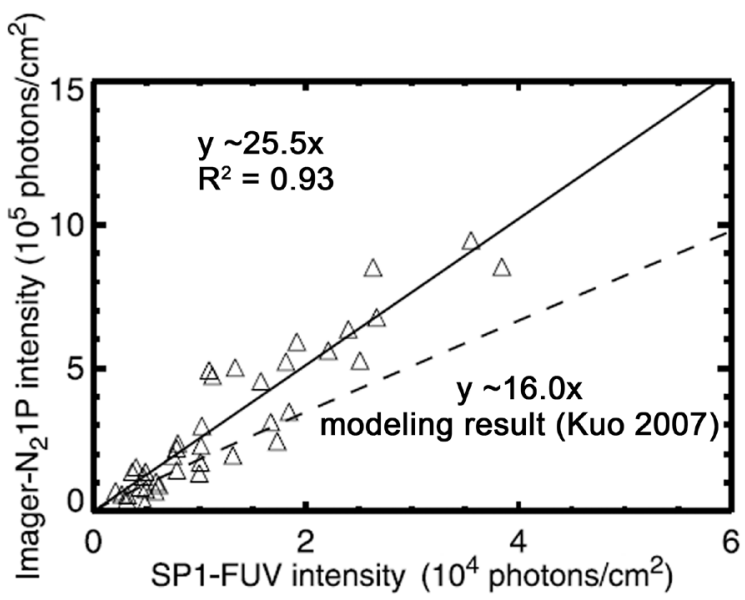

Fig. 9. The linear relations between the ISUAL imager-1PN2 brightness and the ISUAL SP1-FUV intensity for elves. Theoretically, the proportional constant would be sixteen. However in the space environment, the gain of the ISUAL imager and SP are different and the constant becomes 25.5 (Chang et al. 2010). 

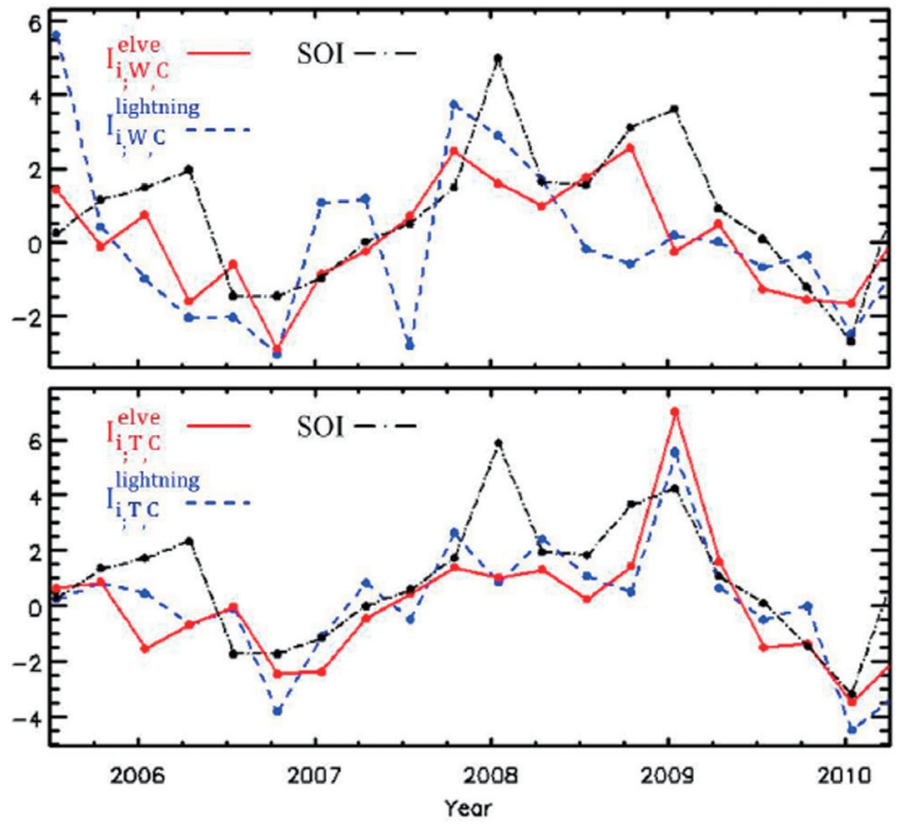

Fig. 10. The occurrence of elves (red line), the occurrence of lightning (blue), and the Southern Oscillation Index (black) for the western Pacificcentral Pacific pair (top) and the Tahiti-central Pacific pair (bottom) (Wu et al. 2012). (Color online only)
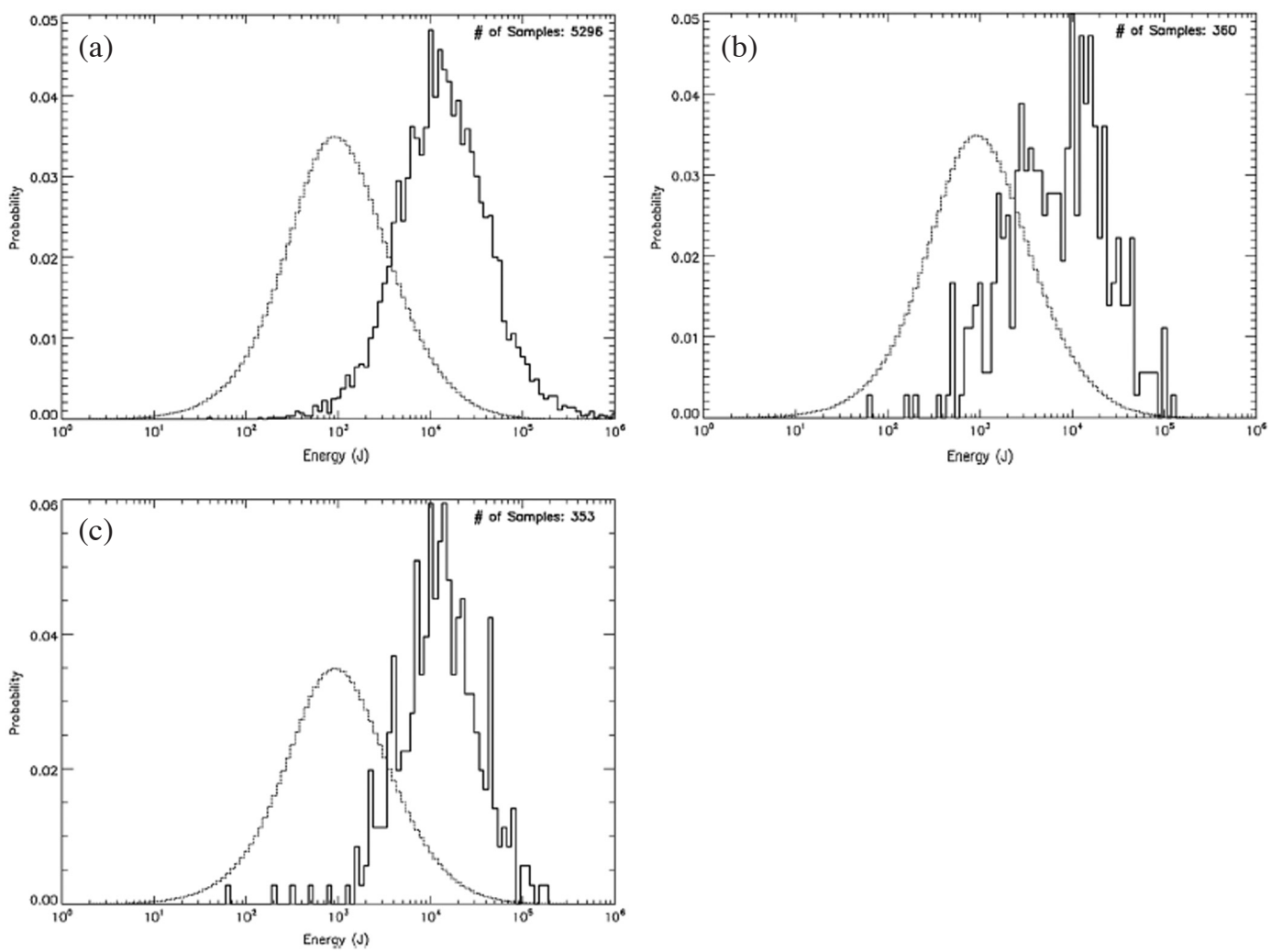

Fig. 11. Energy distribution of TLE-producing strokes for (a) elves, (b) sprites, and (c) halos. The gray curves are the energy distributions of the WWLLN lightning. Apparently, the causative lightning of the ISUAL recorded TLEs are more energetic than the ordinary lightning (Chen et al. 2014). 
strikes. It is also found that elve occurrence rates over the far side of the Earth's limb (behind-the-limb) regions may have been greatly underestimated, partially owing to the lack of triggers to initiate ISUAL recording or the severe atmospheric attenuation to the elves emissions.

\subsection{Nature of Elve-Producing Lightning}

From the analysis of elves recorded in the first four months of ISUAL observation (Frey et al. 2005), it was found that about half of all elves were induced by CG lightning with a beta-type stepped leader. The typical feature of these elves includes an initial brightening in all except the SP1 FUV channels, a slow brightness decrease for the next 2 - 5 milliseconds, and an impulsive signal increase in all channels. We believe that this specific signature could be associated with the initial breakdown with a beta-type stepped leader followed by the bright return stroke of a negative cloud to ground (CG) lightning. In sharp contrast, sprites are often produced by positive cloud to ground lightning (+CG) without the initial breakdown and stepped leader signatures. In the ISUAL data, many sprites are found to be associated with the continuing current and can be delayed up to $100 \mathrm{~ms}$ after the lightning.

\subsection{Electromagnetic Properties of TLE-Producing Lightning}

This study (Chang et al. 2014) used the ISUAL elves occurred within $1500 \mathrm{~km}$ of the NCKU radio-band stations to calibrate the ULF and ELF signals attenuation for elveproducing lightning. The peak sferics value is known to be linearly-dependent upon the peak lightning current. Hence from the recorded sferics, the peak current of the causative lightning can be inferred. There is a functional relationship between the TLE brightness and the peak causative lightning current. For example see Fig. 12, which can also be used to infer the peak TLE-producing lightning current from the TLE brightness. With the calibrated radio-band antennae and the optical TLE imaging system, a thunderstorm system near the Philippines was observed to produce 72 TLEs (57 elves, 4 halos, 2 sprites, and 9 multi-TLE events) on $2 \mathrm{Au}-$ gust 2010. We found that the ULF/VLF ratio can be used to distinguish elve-producing lightning from halo-producing lightning. With this newly acquired information we can examine the hard-to-observe oceanic elves, especially for those located beyond the reach of the optical observation system.

\subsection{Interaction Between Lightning and TLEs}

On 22 July 2007 we recorded more than three dozen TLEs (37 blue jets/starters and one GJ) during a ground observation session. In this multi-band observation, blue jets were found to radiate significant red emissions. However, the blue emissions were completely scattered by the intervening atmosphere. One of the events started off as a blue jet, then after $100 \mathrm{~ms}$, another blue jet occurred at the same location and developed into a GJ. This was the first type-II GJ observed from the ground. From analyzing the lightning optical strikes on the recorded image frames, four notable correlation patterns between jets and lightning were found (Chou et al. 2011). These correlation patterns support that an interaction exists between electrical jets and the conventional cloud lightning. The charge imbalance produced by the cloud lightning could influence the occurrence of jets and the jets could also produce charge imbalances that affect the ensuing lightning activity.

\subsection{Synoptic Factors for the Distribution of TLEs}

Synoptic-scale factors that control the distributions of TLEs over the tropical and winter mid-latitude regions are inferred from ISUAL survey data (Lee et al. 2010). For the tropical regions, the ITCZ (Intertropical Convergence Zone) is the convergence zone for the Hadley cell, mainly formed due to the convergence of the northeasterly trade wind and the southeasterly trade wind from each hemisphere. However, the ITCZ is not solely the result of atmospheric latitudinal circulation; it is also influenced by the longitudinal circulation, the ocean currents and the regional differences in the land-to-ocean ratio. Hence, ITCZ at different longitudinal domains exhibits variations in distribution and migration patterns. Since TLEs are manifestations of deep convections, the ITCZ/SPCZ is expected to be the key factor that affects the tropical TLE distributions and their regional variations should closely match that of the ITCZ/SPCZ (South Pacific Convergence Zone). From the ISUAL TLE data, $84 \%$ of

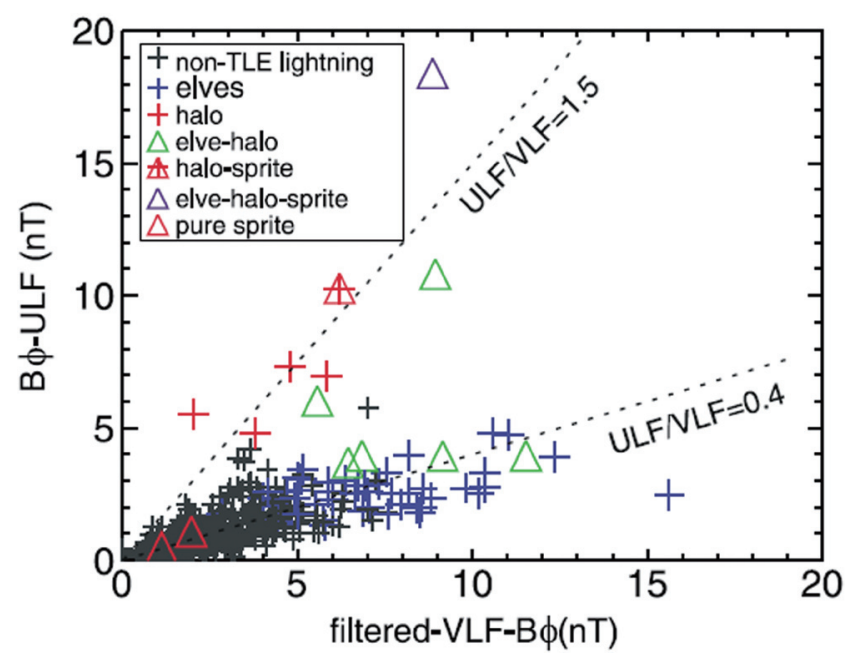

Fig. 12. The ULF/filtered-VLF ratio for the parent lightning of elve, halo, and elves that were accompanied by halo. The dotted-lines are the median values of the ULF/filtered-VLF ratio for the parent lightning of elves and halos (Chang et al. 2014). (Color online only) 
TLEs indeed appear over the ITCZ/SPCZ, and the migrating pattern of TLE distributions in different longitudinal domains closely match those of the ITCZ/SPCZ; Fig. 13.

In the mid-latitude winter regions, the mid-latitude cyclones may contain sufficient energy and instability to invoke convective systems. It was found that most of the winter TLEs occur near the mid-latitude cyclones. In fact, winter TLEs tend to locate near the cold fronts that extend from the mid-latitude cyclone centers. The analyses show that $88 \%$ of the northern winter TLEs and $72 \%$ of the southern winter TLEs are related to the mid-latitude cyclones, where the northern winter TLEs occur mainly over the Pacific Ocean, the Atlantic Ocean, and the Mediterranean Sea. In short, ITCZ located over the tropical regions and the midlatitude cyclones over the winter regions are the synoptic factors that influence the occurrence of TLEs.

\subsection{The TLE Global Distribution and Occurrence Rates}

From the first four years (July 2004 - June 2008) of the ISUAL survey on the FORMOSAT-2 satellite, elves are found to be the most abundant type ( $~ 80 \%$ ) of TLEs, whereas sprites and halos only combine to account for $\sim 20 \%$, defying the impression gained from the ground TLE observation (Chen et al. 2008; Hsu et al. 2009).

Among the TLEs, the distribution of sprites and halos closely resemble that of the CG lightning; see Fig. 14; while $\sim 60 \%$ of elves were found to occur over the oceans. Chen et al. (2008) categorized the Earth's surface into land, coastal and ocean grids according to the grid to land content, a mixture of land and ocean of any proportion, and ocean. After taking the area 1 factor into account, the ratio of elves density between the land/coast and ocean is close to unity. Since elves are induced by lightning and the lightning density over land is about ten times greater than that over the ocean, the land/coast versus ocean ratio is rather stunning.

The detectability of ISUAL TLEs is limited by the background fluctuation of the recorded images. It was found that the ISUAL imager can only detect elves induced by lightning with a peak current of $80 \mathrm{kA}$ or greater (Kuo et al. 2007). The geographic distribution of elves reported in Chen et al. (2008) strongly indicates that the ocean has an exceptionally high proportion of intense lightning. It is conceivable that the smooth ocean surface may have suppressed the occurrence of corona discharges and thus allows the cloud charge of the oceanic thunderstorms to accumulate to a higher level before lightning discharge would occur, compared to their land/coast counterparts. Therefore, for the oceanic lightning with a normal positive-on-negative charge structure, the average peak current for both the negative and the positive lightning is probably higher than that of land/coast lightning.

The ISUAL TLE observation rates for elves, sprites, halos and GJs are found to be 3.62, 0.47, 0.39, and 0.01 events per minute, respectively (Hsu et al. 2009), which is similar to those $\left(3.23,0.50,0.37,0.01\right.$ events minute $\left.^{-1}\right)$ reported in Chen et al. (2008). However, to properly infer the TLE occurrence rates from the detection rates, the correction factors for the observations have to be deduced. If we take the annual occurrence rate of negative CG lightning discharges versus peak current over North America recorded by NLDN from 1989 - 1995 (Wacker and Orville 1999) as the norm, lightning with a peak current exceeding $80 \mathrm{kA}$ account for $\sim 0.8 \%$. Barrington-Leigh and Inan (1999) noted that lightning with a peak current above $60 \mathrm{kA}$ of peak current always had accompanying elves. Since $7 \%$ of lightning has $60 \mathrm{kA}$ or greater peak current, the amount of elves-producing lightning is at least a factor of 10 higher than that producing ISUAL detectable elves. Taking this as the correction factor, Chen et al. (2008) deduced that the elves occurrence rate is $\sim 35$ events minute ${ }^{-1}$.

However, the ISUAL survey zone is limited by the need to protect the sensitive sensors from direct exposure to the Sun. For example, during the northern summer the region above $25^{\circ} \mathrm{N}$ is not observed. Furthermore, the ISUAL survey also contains gaps and reduced detectability regions near the far edges. Considering all of the factors, Hsu et al. (2009) assumed that there is a correction factor of two for the TLE zone under-counting and thus reported an elves occurrence rate of 72 events minute ${ }^{-1}$. Alternatively, using the ISUAL photometric data, Chang et al. (2010) deduced that the ISUAL imager-based survey under-counted elves by an order of magnitude. Applying this correction factor and the abovementioned survey zone correction, the elves occurrence rate again is $\sim 70$ events minute ${ }^{-1}$.

Chen et al. (2008) also examined the relationship between the elves occurrence rate and sea surface temperature (SST), obtained from NODC/RSMAS AVHRR Oceans Pathfinder SST data (Vazquez et al. 1998). The result indicates that the elves production rate is indeed sensitive depending on the SST. The elves occurrence rate increases near-exponentially when the SST exceeds 26 degrees Celsius. Interestingly, the threshold temperature of 26 degrees Celsius is also commonly viewed as a necessary condition for the development of tropical storms, typhoons or hurricanes (Williams and Renno 1993).

\section{THE PHYSICAL CHARACTERISTICS AND THE IMPACTS OF TLES}

\subsection{Electric Field and Electron Energy in Sprites}

Electron energies and the strength of the electric fields in sprites were examined (Kuo et al. 2005). The theoretical peak intensity spectrophotometer channel $2(337.1 \mathrm{~nm})$ and SP3 (391.4 nm) ratio for TLES was computed as a function of the reduced E-field ( $E / N$, where $E$ is the magnitude of the E-field and $N$ is the neutral density). Similarly, the average 
electron energy and the characteristic electron energy were also calculated as a function of the reduced E-field. From the experimental intensity ratio, the reduced E-field for sprites at $40-60 \mathrm{~km}$ is inferred to be $243-443 \mathrm{Td}$. Thus, the electric field strength in a sprite is $2.1-3.7$ times that of the atmosphere breakdown E-field at these altitudes. From the inferred reduced E-field, the average and characteristic electron energies in sprites were found to be in the range of 6.2 -9.2 and $4.5-6.5 \mathrm{eV}$, respectively. The electron energies and the electric field strength in sprites are about two times higher than those previously inferred using the ground observation data. However, they are consistent with the prediction of the sprite streamer model.

\subsection{Electric Field of Diffuse and Streamer Regions in Carrot Sprites}

From the morphological features, sprites can be categorized into carrot and column form sprites. The carrot sprite has a luminous body, tapering towards lower altitudes with

\section{(a) January 2007}

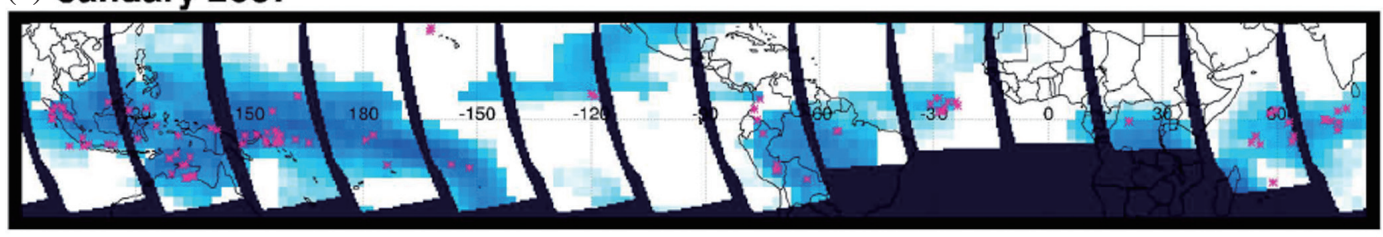

(b) July 2007

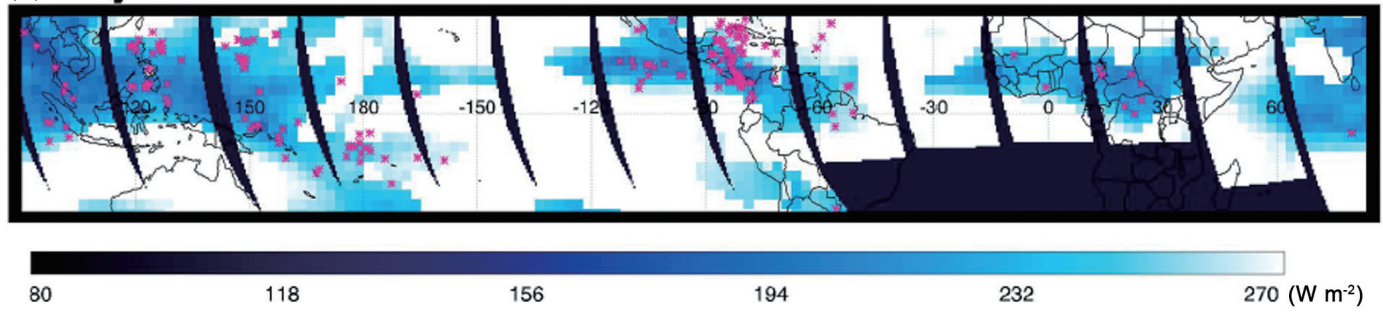

Fig. 13. ISUAL TLEs recorded in January and July 2007 and their distribution relative to the ITCZ. The blue shaded-areas near the equator are the ITCZ, and that south-stretching band in the west Pacific is the SPCZ. The pink symbols mark the locations of the ISUAL TLEs, and the black stripes are the observation gaps of the ISUAL survey. The color bar denotes the outgoing long wave radiation (OLR) at 200-hPa height (Lee et al. 2010). (Color online only)

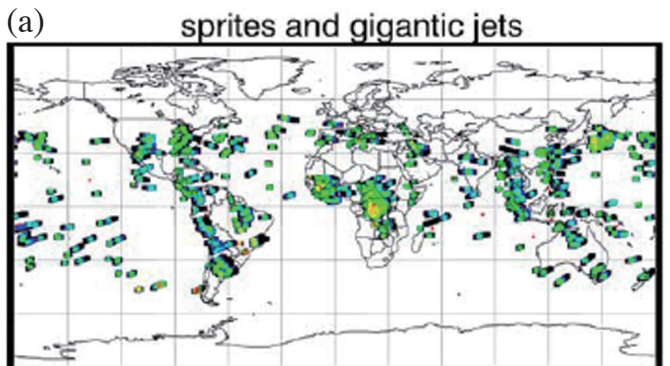

(c)

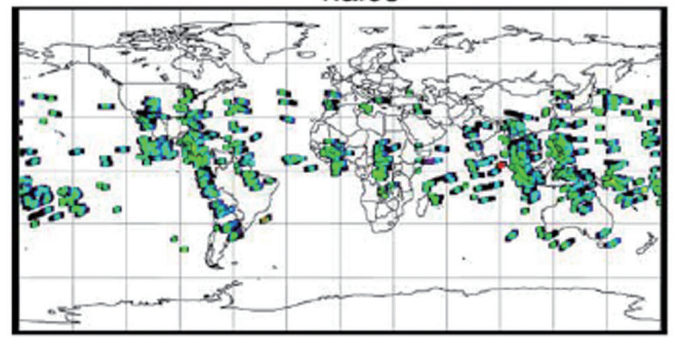

(b) elves

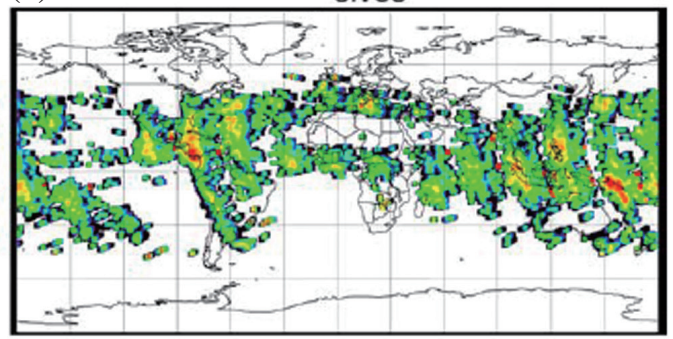

(d)

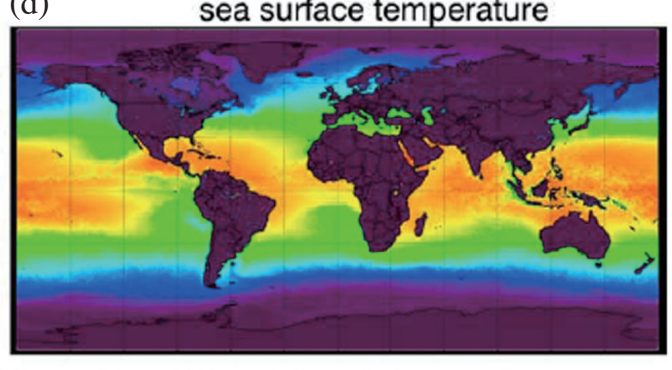

$35\left({ }^{\circ} \mathrm{C}\right)$

Fig. 14. The global distribution of the ISUAL TLEs based on the first three years of satellite survey. The mean sea surface temperature between July 2004 and December 2005 is included for comparison (Chen et al. 2008). (Color online only) 
thin-hairline tendrils and with a diffuse hair region that tops the body. Using the altitudinal resolving data of the ISUAL array photometer, Adachi et al. (2006) computed the blue to the red module AP intensity ratio for twenty carrot sprites and three halos. By assuming all of the recorded emissions were from the electron impact excitation of nitrogen molecules, a transition altitude of $\sim 75 \mathrm{~km}$ was found to separate the upper diffuse and the lower streamer regions; Fig. 15. In the diffuse regions the electric field was inferred to be 0.5 - $0.7 E_{\mathrm{k}}$. These values are consistent with the optical emissions produced without significant ionization. Meanwhile, in the streamer region, the E-fields are $1-2 E_{\mathrm{k}}$, which is substantially lower than those predicted by the streamer model. The discrepancy is probably due to the long-lasting branches and the bead structures in the analyzed sprite events.

\subsection{Luminosity of Sprites and the Charge Moment Change of the Parent Lightning}

In Takahashi et al. (2010), the ISUAL AP and the Tohoku University-ELF data were used to determine the luminosity of fourteen sprites that occurred over the Earth limb and their correlation with the charge moment change of the parent lightning. The 1PN2 (red band) and 2PN2 (blue band) luminosity/energy of these sprites respectively are 176 and $119 \mathrm{~kJ}$. Both band emissions of these sprites are found to be linearly correlated to the charge moment changes of the parent lightning, with a correlation coefficient better than 0.9. The sum of the red and blue band emissions gives the optical energy of the analyzed sprites. Thus, the optical energy (luminosity) and the charge moment change of the parent lightning exhibit a tight linear correlation, as predicted by the QE model; Fig. 16.

\subsection{D-Region Ionization Due to Elves}

The first published journal paper based on the ISUAL space observation data was Mende et al. (2005). Mende et al. (2005) reported on the analysis of a few behind-the-limb elves; elves with the emissions from the causative CG lightning blocked by the solid Earth limb. They showed that the elves contained significant $391.4 \mathrm{~nm} \mathrm{~N}_{2}{ }^{+}$ion emissions, indicating that the generation of elves involved ionization reactions; Fig. 17. They estimated that the elves produced an average electron density of 210 electrons $\mathrm{cm}^{-3}$ over a large (165 km diameter) circular region having an assumed $10 \mathrm{~km}$ altitude extent; roughly increasing the local free electron density by $100 \%$. Using a theoretical TLE emission model, the electron energy distribution and the ratios between the ISUAL SP1 (LBH) as well as the ISUAL SP3 $\left(1 \mathrm{NN}_{2}{ }^{+}\right)$and the SP2 $\left(2 \mathrm{PN}_{2}\right)$ can be computed as a function of the reduced electric field. From the ratio computed using the ISUAL SP3 and SP2 readings, the reduced electric field in these elves is inferred to be greater than $200 \mathrm{Td}$, which is substantially higher than the breakdown E-field of $128 \mathrm{Td}$ and is indeed capable of producing the ionization emissions.

\subsection{Morphologies and Photometric Emissions of Elves}

Kuo et al. (2007) used the elves emission model to perform detailed numerical simulations on the spatial and temporal evolution of elves. The simulated elve morphologies are in excellent agreement with the before-the-limb, at-thelimb and behind-the limb elves observed by ISUAL; Fig. 18 . The relationship between the ISUAL imager photon counts and the peak current of the elve-producing lightning is also computed and validated by the US National Lightning Detection Network recorded elve-associated CG lightning.

\subsection{Charge Impact of Elves to the Mesospheric Electron Density}

By analyzing consecutive sprites that occurred at the same location, we learned that the upper atmospheric plasma in a sprite could persist for 5 minutes or even longer (Y. J. Lee 2001, personal communication). Since the elve occurrence rate is an order of magnitude higher than those for the other TLEs (Chen et al. 2008), elves may significantly impact the charge content of the mesosphere. The mean nighttime free electron density at $80-90 \mathrm{~km}$ altitudes is $90 \mathrm{~cm}^{-3}$ according to the International Reference Ionosphere (IRI) model (Bilitza et al. 2014). Mende et al. (2005) reported that the free electron density inside an elves can be as high as $210 \mathrm{~cm}^{-3}$, roughly twice that of the ambient density. Therefore, elves and other TLEs are expected to disturb radio communications and their influence on VLF transmitters has indeed been detected (Mika et al. 2006; Inan et al. 2010 and the references therein).

If elves are taken as cylindrical in shape with a

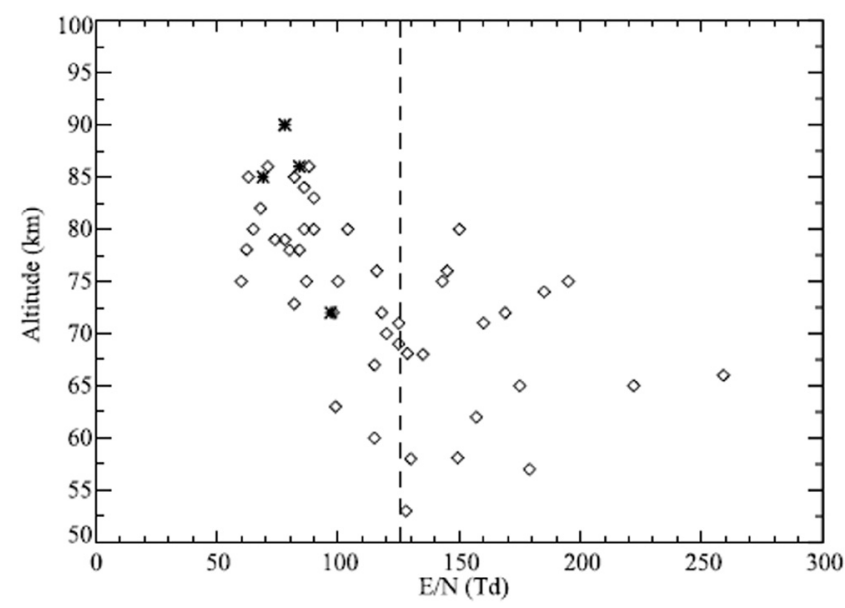

Fig. 15. Altitudinal variation of the reduced E-field $\mathrm{E} / \mathrm{N}$ for sprites (diamonds) and halos (asterisks). The convectional breakdown E-field in air (128 Td) is denoted by the dashed line (Adachi et al. 2006). 


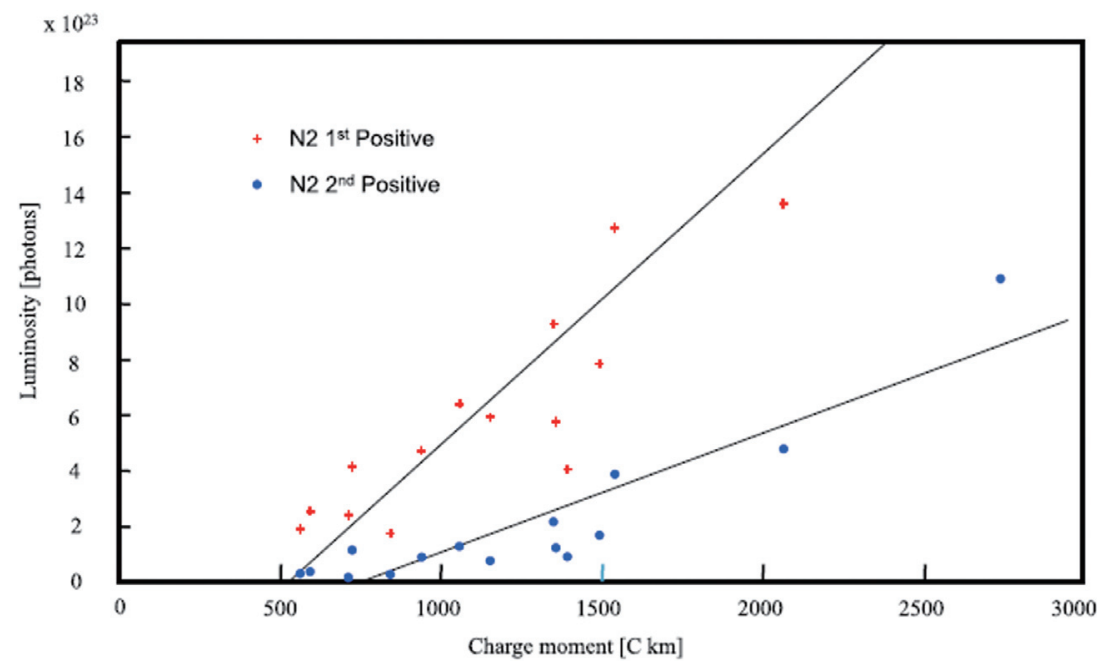

Fig. 16. Correlation between the luminosity of sprites and the charge moment change of the parent lightning. Red dots are the emitted photons in the $1 \mathrm{PN}_{2}$ band and the blue dots denote the $2 \mathrm{PN}_{2}$ band (Takahashi et al. 2010). (Color online only)

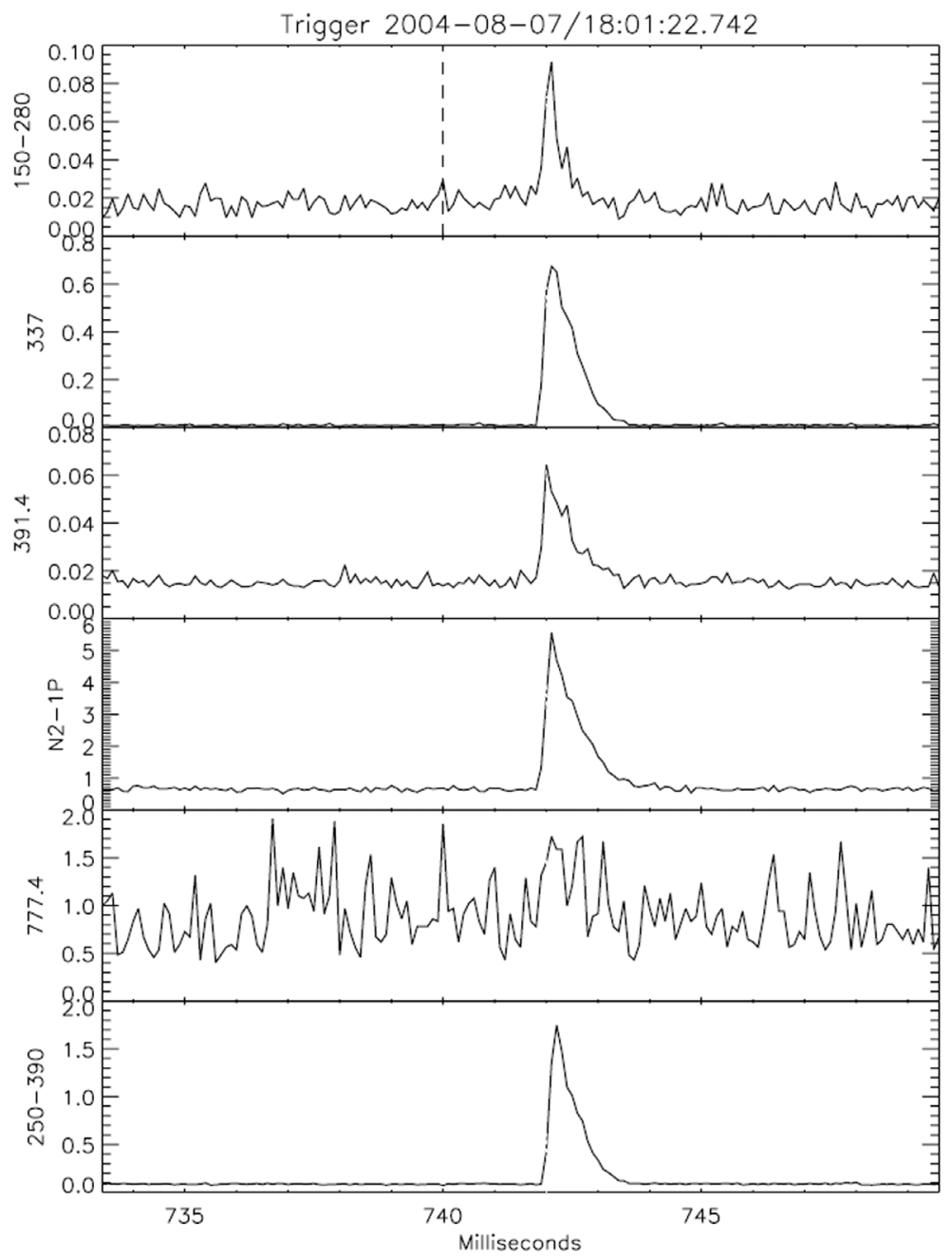

Fig. 17. The representative spectrophotometric data for an elves recorded on 7 August 2004. The SP3 (391.4 nm) trace indicates the generation of elves involves ionization processes (Mende et al. 2005). 
(a)

2004/08/13 09:21:25.787

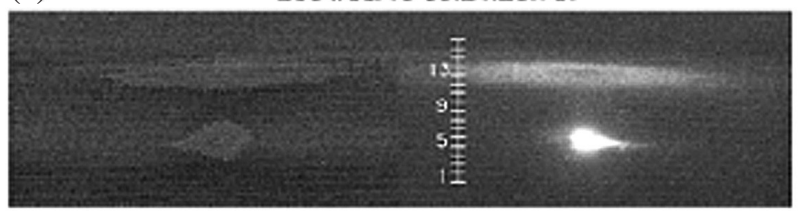

$2004 / 07 / 0614: 42: 06.266$
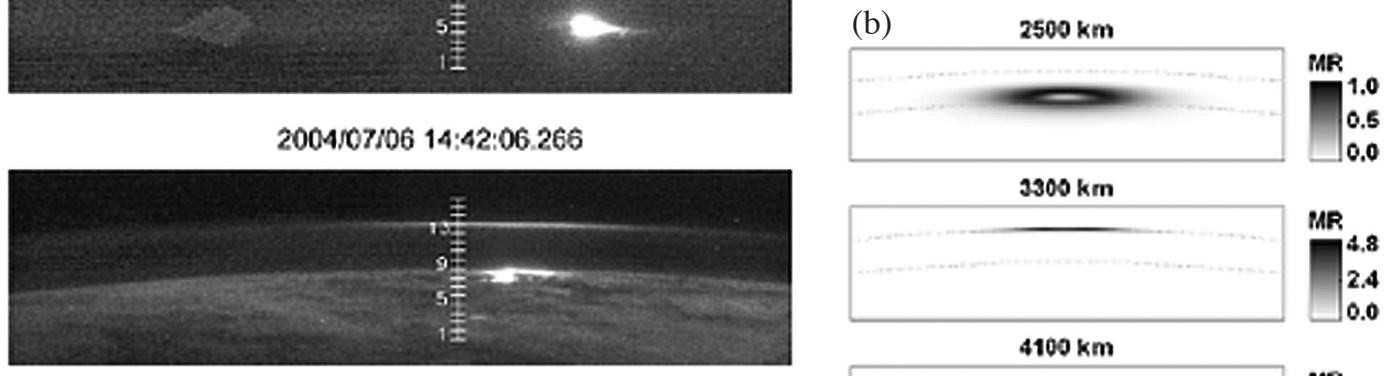

2004/08/07 18:01:22.742
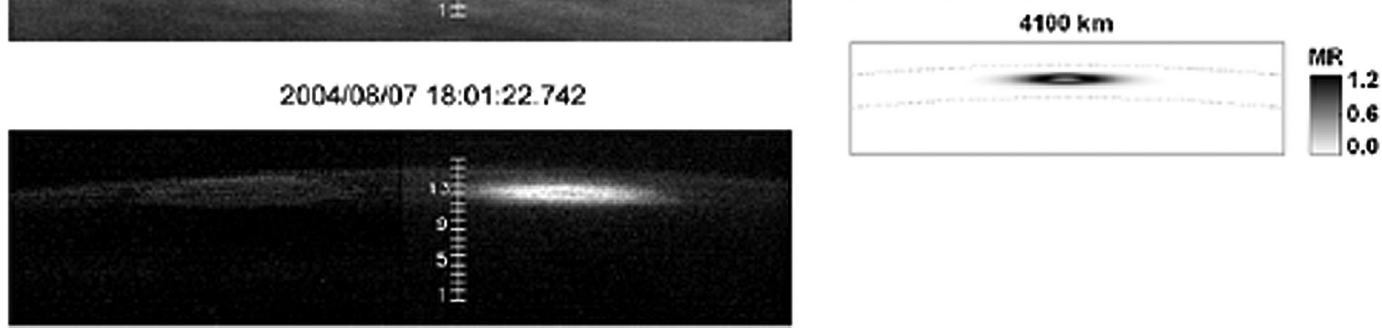

Fig. 18. (a) Left panels viewing from top to down, before-the-limb, at-the-limb, and behind-the-limb elves as recorded by the ISUAL imager. (b) Right panels viewing from top to down. The corresponding modeled elves (Kuo et al. 2007).

diameter of $\sim 170 \mathrm{~km}$ and spanning the vertical height between 80 and $90 \mathrm{~km}$, the volume taken up by the elves is then $\sim 2.3 \times 10^{5} \mathrm{~km}^{3}$. For the Caribbean elve hot zone, the elve occurrence rate is 3.0 events minute ${ }^{-1}\left(r_{\text {local }}\right)$ and the lifetime of the plasma clouds is assumed to be 2 minutes $\left(l_{\text {lifetime }}\right)$. The fractional local electron density change is defined as $f=\Delta n_{e} \cdot V_{\text {elve }} \cdot r_{\text {local }} \cdot l_{\text {lifetime }} / n_{e} \cdot V_{\text {volume }} \approx 10 \%$; where $n_{\mathrm{e}}$ is the mean nighttime free electron density at $80-90 \mathrm{~km}$ altitudes, $\Delta n_{e}$ is the free electron density increase in an elve, and $V_{\text {local }}$ is the volume $\left(1.23 \times 10^{7} \mathrm{~km}^{3}\right)$ between 80 and $90 \mathrm{~km}$ above the Caribbean region. Hence the free electron content for the mesosphere over the Caribbean region at most can be elevated by $\sim 10 \%$ (Chen et al. 2008; Hsu et al. 2009).

\subsection{Energy Impact of TLEs to the Upper Atmosphere}

TLEs are the radiative manifestation of the electrodynamic coupling between the troposphere and the upper atmosphere. Thus elucidating the TLE energy deposition in the upper atmosphere is a way to quantify this coupling as well as the role of TLEs in the Earth's environment. An in-depth presentation on how to find the TLE energy deposition can be found in Kuo et al. (2008). Here we only provide a simple conceptual outline. In the first step, the theoretical spectra of the major molecular nitrogen band systems, $1 \mathrm{PN}_{2}, 2 \mathrm{PN}_{2}, \mathrm{~N} 2-\mathrm{LBH}$, and $1 \mathrm{NN}_{2}{ }^{+}$in TLEs have to be computed. Before finding the energy in a TLE event with a known event distance, the lightning contamination and the atmospheric absorption in the ISUAL data need to be corrected. Assuming the TLE emissions are isotropic emissions, the SP time-integrated photon intensity can be used to infer the number of photons in a specified SP band from a TLE. The SP measured photon numbers are used to deduce the total excited numbers for the $1 \mathrm{PN}_{2}, 2 \mathrm{PN}_{2}$, N2$\mathrm{LBH}$, and $1 \mathrm{NN}_{2}{ }^{+}$bands using the known band contributions. After further multiplying the excited number in each band with its threshold energy, the energy associated with each molecular nitrogen emission band can be obtained. The estimated energy for a given TLE can be obtained by summing all of the bands.

Kuo et al. (2008) analyzed 1415 ISUAL TLEs and found that the average energy deposited by sprites, halos, and ELVEs are 22, 14, and $19 \mathrm{MJ}$, respectively. With the updated ISUALTLE global occurrence rates (Hsu et al. 2009), the energy deposition rates are inferred to be 22,52 , and $1370 \mathrm{MJ}^{\text {minute }}{ }^{-1}$ for sprites, halos, and elves.

\subsection{Discharge Processes, Electric Field, and Electron Energy in Gigantic Jets}

Spectral ratio analysis (SP2/SP3) for the fully-developed jet in GJs yields a reduced E field of $400-655 \mathrm{Td}$ and average electron energy of $8.5-12.3 \mathrm{eV}$ (Kuo et al. 2009). Evidently, these values are higher than those in the sprites (Kuo et al. 2005) but in line with those predicted by streamer models, which indicate that there are streamer-tips in the fully developed jets. The GJs studied here all feature two closely separated photometric peaks; Fig. 19. The first peak is from the fully-developed jet that steadily surges from the cloud top $(\sim 20 \mathrm{~km})$ to the lower ionosphere at $\sim 90 \mathrm{~km}$. The second photometric peak that appears $\sim 1 \mathrm{~ms}$ after the first peak may be from a current wave or potential wave-enhanced 


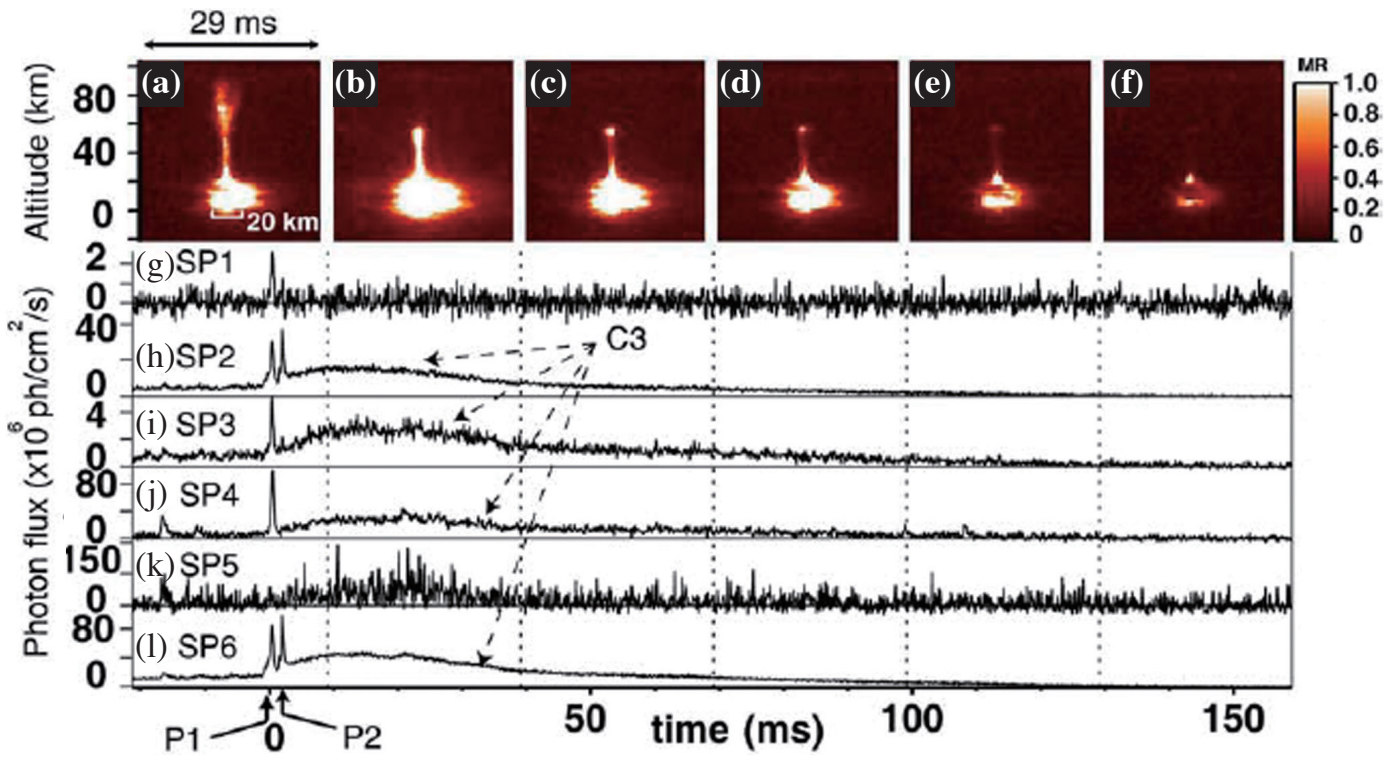

Fig. 19. (a) - (g) Image sequence and (f) - (l) photometric signals for the ISUAL GJ on 1 October 2005. The photometric traces indicate this GJ have two photometric peaks: the first peak (P1) was from the fully-developed jet and the second peak (P2) was from a return-stroke-like process (Kuo et al. 2009). (Color online only)

emissions that originate at an altitude of $\sim 50 \mathrm{~km}$ and develop toward the cloud top. It is possible that the fully-developed jet becomes an extension of the local ionosphere and forms a lowered ionosphere boundary. As the attachment processes remove the charges, the boundary of the local ionosphere moves up. The current in the channel persists and its contact point with the ionosphere also moves upward, that produces the upward surging trailing jets. Imager and photometer data indicate that the lightning activity associated with the GJs is likely in-cloud, and thus the initiation of the GJs is not directly associated with CG discharges. From the GJ dynamic process development, GJs behave like the electric discharge between a thundercloud and the ionosphere, which can be viewed as the counterpart of conventional CG discharges

\section{CONCLUSION}

After more than a decade of TLE surveys on the FORMOSAT2 satellite, the ISUAL mission has yielded a unique and valuable dataset. From analyzing the ISUAL TLE data, some important insights on these intriguing cloud-top phenomena have been obtained, including their occurrence, their global distributions, their occurrence rates, their characteristics, some salient properties of the TLE-producing lightning, and the impacts of TLEs on the upper atmosphere. More specific findings of the ISUAL mission are:

From the ISUAL survey, elves were found to be the dominate TLE species, which is a remarkable departure from sprites being the major species as determined from ground TLEs observations (Chen et al. 2008).

The lightning occurrence density over land and over ocean is known to be $\sim 10: 1$ (Rakov and Uman 2003). However, ISUAL found that $60 \%$ of the lightning-induced elves appear over the ocean (Chen et al. 2008). This is an indication that elve-inducting high peak current lightning is more prevalent over the ocean, probably due to the diminishing number of corona discharge sources. Also from the ISUAL survey, the synoptic factor that controls the TLE distribution in the tropical regions appears to be ITCZ/SPCZ, while during the winter mid-latitudinal regions it is the winter cyclones (Lee et al. 2010).

From the ISUAL spectrophotometric data, the type-I GJs were found to be the true counterpart of the convectional CG lightning (Kuo et al. 2009). After the GJ contacted the lower ionospheric boundary, the highly-ionized discharge channel effectively lowers the local ionospheric boundary to $\sim 50 \mathrm{~km}$. From the lowered boundary, a return-stroke like process develops toward the cloud-top. With the attachment processes removing the charges, the boundary of the local ionosphere gradually recovers and the manifestation is an upward-surging trailing jet.

From the ISUAL TLE data, Chen et al. (2008) inferred the global detection rates are $3.23,0.50,0.39$, and 0.01 events per minute for elves, sprites, halos, and GJs, respectively. After taking the correction factors into account, the global occurrence rates are respectively inferred to be 72 , 3.7 , and $\sim 1$ events minute ${ }^{-1}$ for elves, halos, and sprites (Hsu et al. 2009).

From the spectrophometric data, Mende et al. (2005) had inferred that the electron density in elves can increase by $\sim 100 \%$ above the ambient. If we further consider the elves occurrence rate over the Caribbean region, the free 
electron density over this region can be elevated by $\sim 10 \%$. Also from the spectrophometric data, the average energy deposition per event for sprites, halos and elves are respectively deduced to be 22, 14 and $19 \mathrm{MJ}$ (Kuo et al. 2008). After factoring in the TLE global occurrence rates, the energy deposition rates are computed to be 22,52 , and $1370 \mathrm{MJ}$ minute $^{-1}$ for sprites, halos, and elves (Hsu et al. 2009).

Our understanding of these relatively late discovered transient events is far from complete. For example, as of December of 2015, the TLE events recorded by the ISUAL include nearly five thousands blue jets. The majority of them actually occurred successively within the two-minute observation window of the ISUAL sensors. Why should they appear in quick succession and in the same cloud-top area? Currently, we suspect that it may have something to do with the shearing winds near the active thunderstorms. Further study is clearly needed to resolve this puzzle. In addition, there are numerous open questions of TLEs and here are some of them:

(1) Do cosmic rays, gravity waves, meteors and meteoric dust influence TLE initiation (Lyons 2006 and the references therein)?

(2) Do the characteristics of TLEs depend on the type of the parent thunderstorm, geographic region, season, and lightning type (Price 2006)?

(3) The top of sprites and GJs often terminates near $90 \mathrm{~km}$ while exhibits some variations from event to event. Can the height of TLEs be used as an indicator of the local ionosphere boundary or even the sporadic D-layer of the night hemisphere?

(4) What are the roles played by TLEs in the coupling of different atmospheric layers?

(5) Can the electromagnetic wave launched by TLEs penetrate and propagate in the ionosphere?

(6) TLEs are often said to be parts of the global electric circuit. However, a quantitative assessment is still lacking. Along the same line, exactly how important TLEs are to the Earth's environment?

Besides scientific explorations, the ISUAL team members at the NCKU are also responsible for the day to day planning and operation of the ISUAL experiment. This hand-on experience is very valuable and the team could be an important consulting source for the other teams that are planning or operating a satellite TLE payload. Among the experience gained from executing the ISUAL mission, the detection and the time keeping of TLEs are particularly noteworthy. The far ultra-violet emission $(150 \mathrm{~nm}-290 \mathrm{~nm})$ has proven to be a signature emission for the occurrence of TLEs; for an example, see Chang et al. 2010. Future space missions may consider incorporating a FUV sensor for TLE detection. Moreover, a high precision $\left(\sim 10^{-6} \mathrm{sec}\right)$ onboard clock would be important equipment for finding the TLEassociated electromagnetic signals detected by the ground stations.
Acknowledgements The authors would like to acknowledge the financial support from the Ministry of Science and Technology and National Space Organization in Taiwan through the years. Logistical support from the NCU-Lulin Observatory, Cingcao Elementary School, Jin-cheng Junior High School, NPU, NTTU, and Tu-Cheng High School are greatly appreciated. The authors are grateful to J. K. Chou, S. C. Chang, Y. J. Wu, and K. M. Peng for their fruitful discussions.

\section{REFERENCES}

Adachi, T., H. Fukunishi, Y. Takahashi, Y.Hiraki, R. R.Hsu, H. T. Su, A. B. Chen, S. B. Mende, H. U. Frey, and L. C. Lee, 2006: Electric field transition between the diffuse and streamer regions of sprites estimated from ISUAL/ array photometer measurements. Geophys. Res. Lett., 33, L17803, doi: 10.1029/2006GL026495. [Link]

Barrington-Leigh, C.P. and U. S. Inan, 1999: Elves triggered by positive and negative lightning discharges. Geophys. Res. Lett., 26, 683-686, doi: 10.1029/1999GL900059. [Link]

Barrington-Leigh, C. P., U. S. Inan, and M. Stanley, 2001: Identification of sprites and elves with intensified video and broadband array photometry. J. Geophys. Res., 106, 1741-1750, doi: 10.1029/2000JA000073. [Link]

Bilitza, D., D. Altadill, Y. Zhang, C. Mertens, V. Truhlik, P. Richards, L. A. McKinnell, and B. Reinisch, 2014: The International Reference Ionosphere 2012 - a model of international collaboration. J. Space Weather Space Clim., 4, A07, doi: 10.1051/swsc/2014004. [Link]

Chang, S. C., C. L. Kuo, L. J. Lee, A. B. Chen, H. T. Su, R. R. Hsu, H. U. Frey, S. B. Mende, Y. Takahashi, and L. C. Lee, 2010: ISUAL far-ultraviolet events, elves, and lightning current. J. Geophys. Res., 115, A00E46, doi: 10.1029/2009JA014861. [Link]

Chang, S. C., R. R. Hsu, S. M. Huang, H. T. Su, C. L. Kuo, J. K. Chou, L. J. Lee, Y. J. Wu, and A. B. Chen, 2014: Characteristics of TLE-producing lightning in a coastal thunderstorm. J. Geophys. Res., 119, 9303-9320, doi: 10.1002/2014JA019819. [Link]

Chen, A. B., C. L. Kuo, Y. J. Lee, H. T. Su, R. R. Hsu, J. L. Chern, H. U. Frey, S. B. Mende, Y. Takahashi, H. Fukunishi, Y. S. Chang, T. Y. Liu, and L. C. Lee, 2008: Global distributions and occurrence rates of transient luminous events. J. Geophys. Res., 113, A08306, doi: 10.1029/2008JA013101. [Link]

Chen, A. B. C., H. T. Su, and R. R. Hsu, 2014: Energetics and geographic distribution of elve-producing discharges. J. Geophys. Res., 119, 1381-1391, doi: 10.1002/2013JA019470. [Link]

Chern, J. L., R. R. Hsu, H. T. Su, S. B. Mende, H. Fukunishi, Y. Takahashi, and L. C. Lee, 2003: Global survey of upper atmospheric transient luminous events on 
the ROCSAT-2 satellite. J. Atmos. Sol.-Terr. Phys., 65, 647-659, doi: 10.1016/S1364-6826(02)00317-6. [Link]

Chou, J. K., C. L. Kuo, L. Y. Tsai, A. B. Chen, H. T. Su, R. R. Hsu, S. A. Cummer, J. Li, H. U. Frey, S. B. Mende, Y. Takahashi, and L. C. Lee, 2010: Gigantic jets with negative and positive polarity streamers. J. Geophys. Res., 115, A00E45, doi: 10.1029/2009JA014831. [Link]

Chou, J. K., L. Y. Tsai, C. L. Kuo, Y. J. Lee, C. M. Chen, A. B. Chen, H. T. Su, R. R. Hsu, P. L. Chang, and L. C. Lee, 2011: Optical emissions and behaviors of the blue starters, blue jets, and gigantic jets observed in the Taiwan transient luminous event ground campaign.J.Geophys. Res., 116, A07301, doi: 10.1029/2010JA016162. [Link]

Franz, R. C., R. J. Nemzek, and J. R. Winckler, 1990: Television image of a large upward electrical discharge above a thunderstorm system. Science, 249, 48-51, doi: 10.1126/science. 249.4964.48. [Link]

Frey, H. U., S. B. Mende, S. A. Cummer, A. B. Chen, R. R. Hsu, H. T. Su, Y. S. Chang, T. Adachi, H. Fukunishi, and Y. Takahashi, 2005: Beta-type stepped leader of elve-producing lightning. Geophys. Res. Lett., 32, L13824, doi: 10.1029/2005GL023080. [Link]

Fukunishi, H., Y. Takahashi, M. Kubota, K. Sakanoi, U. S. Inan, and W. A. Lyons, 1996: Elves: Lightninginduced transient luminous events in the lower ionosphere. Geophys. Res. Lett., 23, 2157-2160, doi: 10.1029/96GL01979. [Link]

Hsu, R. R., H. T. Su, A. B. Chen, L. C. Lee, M. Asfur, C. Price, and Y. Yair, 2003: Transient luminous events in the vicinity of Taiwan. J. Atmos. Sol.-Terr. Phys., 65, 561-566, doi: 10.1016/S1364-6826(02)00320-6. [Link]

Hsu, R. R., A. B. Chen, C. L. Kuo, H. T. Su, H. Frey, S. Mende, Y. Takahashi, and L. C. Lee, 2009: On the global occurrence and impacts of transient luminous events (TLEs). AIP Conf. Proc., 1118, 99-107, doi: 10.1063/1.3137720. [Link]

Huang, S. M., R. R. Hsu, L. J. Lee, H. T. Su, C. L. Kuo, C. C. Wu, J. K. Chou, S. C. Chang, Y. J. Wu, and A. B. Chen, 2012: Optical and radio signatures of negative gigantic jets: Cases from Typhoon Lionrock (2010). J. Geophys. Res., 117, A08307, doi: 10.1029/2012JA017600. [Link]

Inan, U. S., S. A. Cummer, and R. A. Marshall, 2010: A survey of ELF and VLF research on lightning-ionosphere interactions and causative discharges. J. Geophys. Res., 115, A00E36, doi: 10.1029/2009JA014775. [Link]

Kuo, C. L., R. R. Hsu, A. B. Chen, H. T. Su, L. C. Lee, S. B. Mende, H. U. Frey, H. Fukunishi, and Y. Takahashi, 2005: Electric fields and electron energies inferred from the ISUAL recorded sprites. Geophys. Res. Lett.,
32, L19103, doi: 10.1029/2005GL023389. [Link]

Kuo, C. L., A. B. Chen, Y. J. Lee, L. Y. Tsai, R. K. Chou, R. R. Hsu, H. T. Su, L. C. Lee, S. A. Cummer, H. U. Frey, S. B. Mende, Y. Takahashi, and H. Fukunishi, 2007: Modeling elves observed by FORMOSAT-2 satellite. J. Geophys. Res., 112, A11312, doi: 10.1029/2007JA012407. [Link]

Kuo, C. L., A. B. Chen, J. K. Chou, L. Y. Tsai, R. R. Hsu, H. T. Su, H. U. Frey, S. B. Mende, Y. Takahashi, and L. C. Lee, 2008: Radiative emission and energy deposition in transient luminous events. J. Phys. D: Appl. Phys., 41, 234014, doi: 10.1088/0022-3727/41/23/234014. [Link]

Kuo, C. L., J. K. Chou, L. Y. Tsai, A. B. Chen, H. T. Su, R. R. Hsu, S. A. Cummer, H. U. Frey, S. B. Mende, Y. Takahashi, and L. C. Lee, 2009: Discharge processes, electric field, and electron energy in ISUAL-recorded gigantic jets. J. Geophys. Res., 114, A04314, doi: 10.1029/2008JA013791. [Link]

Lee, L. J., A. B. Chen, S. C. Chang, C. L. Kuo, H. T. Su, R. R. Hsu, C. C. Wu, P. H. Lin, H. U. Frey, S. B. Mende, Y. Takahashi, and L. C. Lee, 2010: Controlling synoptic-scale factors for the distribution of transient luminous events. J. Geophys. Res., 115, A00E54, doi: 10.1029/2009JA014823. [Link]

Lee, L. J., S. M. Huang, J. K. Chou, C. L. Kuo, A. B. Chen, H. T. Su, R. R. Hsu, H. U. Frey, Y. Takahashi, and L. C. Lee, 2012: Characteristics and generation of secondary jets and secondary gigantic jets. J. Geophys. Res., 117, A06317, doi: 10.1029/2011JA017443. [Link]

Lee, L. J., R. R. Hsu, H. T. Su, S. M. Huang, J. K. Chou, C. L. Kuo, S. C. Chang, Y. J. Wu, A. B. Chen, H. U. Frey, Y. Takahashi, and L. C. Lee, 2013: Secondary gigantic jets as possible inducers of sprites. Geophys. Res. Lett., 40, 1462-1467, doi: 10.1002/grl.50300. [Link]

Lyons, W. A., 2006: The meteorology of transient luminous events - an introduction and overview. In: Fullekrug, M., E. A. Mareev, and M. J. Rycroft (Eds.), Sprites, Elves and Intense Lightning Discharges, NATO Science Series II: Mathematics, Physics and Chemistry, Vol. 225, 19-56, Springer, Dordrecht, doi: 10.1007/14020-4629-4_2. [Link]

Lyons, W. A., R. A. Armstrong, E. A. Bering III, and E. R. Williams, 2000: The hundred year hunt for the sprite. Eos, Trans., AGU, 81, 373-377, doi: 10.1029/00EO00278. [Link]

Mende, S. B., H. U. Frey, R. R. Hsu, H. T. Su, A. B. Chen, L. C. Lee, D. D. Sentman, Y. Takahashi, and H. Fukunishi, 2005: $D$ region ionization by lightning-induced electromagnetic pulses. J. Geophys. Res., 110, A11312, doi: 10.1029/2005JA011064. [Link]

Mika, A., C. Haldoupis, T. Neubert, H. T. Su, R. R. Hsu, R. J. Steiner, and R. A. Marshall, 2006: Early VLF perturbations observed in association with elves. 
Ann. Geophys., 24, 2179-2189, doi: 10.5194/angeo-24-2179-2006. [Link]

Pasko, V. P., 2010: Recent advances in theory of transient luminous events. J. Geophys. Res., 115, A00E35, doi: 10.1029/2009JA014860. [Link]

Pasko, V. P., U. S. Inan, T. F. Bell, and Y. N. Taranenko, 1997: Sprites produced by quasi-electrostatic heating and ionization in the lower ionosphere. J. Geophys. Res., 102, 4529-4561, doi: 10.1029/96JA03528. [Link]

Pasko, V. P., M. A. Stanley, J. D. Mathews, U. S. Inan, and T. G. Wood, 2002: Electrical discharge from a thundercloud top to the lower ionosphere. Nature, 416, 152-154, doi: 10.1038/416152a. [Link]

Pasko, V. P., Y. Yair, and C. L. Kuo, 2012: Lightning related transient luminous events at high altitude in the Earth's atmosphere: Phenomenology, mechanisms and effects. Space Sci. Rev., 168, 475-516, doi: 10.1007/ s11214-011-9813-9. [Link]

Price, C., 2006: Global thunderstorm activity. In: Füllekrug, M., E. A. Mareev, and M. J. Rycroft, (Eds.) Sprites, Elves and Intense Lightning Discharges, NATO Science Series II: Mathematics, Physics and Chemistry, Vol. 225, 85-99, Springer, Dordrecht, doi: 10.1007/14020-4629-4_4. [Link]

Rakov, V. A. and M. A. Uman, 2003: Lightning: Physics and Effects, Cambridge Univ. Press, Cambridge, U.K., ISBN 0-521-58327-6.

Sentman, D. D. and E. M. Wescott, 1993: Observations of upper atmospheric optical flashes recorded from an aircraft. Geophys. Res. Lett., 20, 2857-2860, doi: 10.1029/93GL02998. [Link]

Su, H. T., R. R. Hsu, A. B. Chen, Y. J. Lee, and L. C. Lee, 2002: Observation of sprites over the Asian continent and over oceans around Taiwan. Geophys. Res. Lett., 29, 3-1-3-4, doi: 10.1029/2001GL013737. [Link]

Su, H. T., R. R. Hsu, A. B. Chen, Y. C. Wang, W. S. Hsiao, W. C. Lai, L. C. Lee, M. Sato, and H. Fukunishi, 2003: Gigantic jets between a thundercloud and the ionosphere. Nature, 423, 974-976, doi: 10.1038/nature01759. [Link]
Takahashi, Y., A. Yoshida, M. Sato, T. Adachi, S. Kondo, R. R. Hsu, H. T. Su, A. B. Chen, S. B. Mende, H. U. Frey, and L. C. Lee, 2010: Absolute optical energy of sprites and its relationship to charge moment of parent lightning discharge based on measurement by ISUAL/AP. J. Geophys. Res., 115, A00E55, doi: 10.1029/2009JA014814. [Link]

Van der Velde, O. A., J. Bór, J. Li, S. A. Cummer, E. Arnone, F. Zanotti, M. Füllekrug, C. Haldoupis, S. NaitAmor, and T. Farges, 2010: Multi-instrumental observations of a positive gigantic jet produced by a winter thunderstorm in Europe. J. Geophys. Res., 115, D24301, doi: 10.1029/2010JD014442. [Link]

Vazquez, J., K. Perry, and K. Kilpatrick, 1998: NOAA/ NASA AVHRR Oceans Pathfinder Sea Surface Temperature Data Set User's Reference Manual Version 4.0. JPL Publication D-14070.

Wacker, R. S. and R. E. Orville, 1999: Changes in measured lightning flash count and return stroke peak current after the 1994 U.S. National Lightning Detection Network upgrade: 1. Observations. J. Geophys. Res., 104, 2151-2157, doi: 10.1029/1998JD200060. [Link]

Wescott, E. M., D. Sentman, D. Osborne, D. Hampton, and M. Heavner, 1995: Preliminary results from the Sprites94 aircraft campaign: 2. Blue jets. Geophys. Res. Lett., 22, 1209-1212, doi: 10.1029/95GL00582. [Link]

Williams,E.R.,2010: Origin and context of C.T.R. Wilson's ideas on electron runaway in thunderclouds. J. Geophys. Res., 115, A00E50, doi: 10.1029/2009JA014581. [Link]

Williams, E. R. and N. Renno, 1993: An analysis of the conditional instability of the tropical atmosphere. Mon. Weather Rev., 121, 21-36, doi: 10.1175/1520-0493(19 93) $121<0021$ :AAOTCI>2.0.CO;2. [Link]

Wu, Y. J., A. B. Chen, H. H. Hsu, J. K. Chou, S. C. Chang, L. J. Lee, Y. J. Lee, H. T. Su, C. L. Kuo, R. R. Hsu, H. U. Frey, S. B. Mende, Y. Takahashi, and L. C. Lee, 2012: Occurrence of elves and lightning during El Niño and La Niña. Geophys. Res. Lett., 39, L03106, doi: 10.1029/2011GL049831. [Link] 